\title{
Existence and Convergence Properties of Physical Measures for Certain Dynamical Systems with Holes
}

Henk Bruin

Mark Demers

Fairfield University, mdemers@fairfield.edu

Ian Melbourne

Follow this and additional works at: https://digitalcommons.fairfield.edu/mathandcomputersciencefacultypubs

Copyright 2010 by Cambridge University Press. Original published version can be found at DOI: $10.1017 / \mathrm{S} 0143385709000200$

\section{Peer Reviewed}

\section{Repository Citation}

Bruin, Henk; Demers, Mark; and Melbourne, lan, "Existence and Convergence Properties of Physical Measures for Certain Dynamical Systems with Holes" (2010). Mathematics Faculty Publications. 10. https://digitalcommons.fairfield.edu/mathandcomputerscience-facultypubs/10

\section{Published Citation}

H. Bruin, M. Demers, and I. Melbourne. Existence and Convergence Properties of Physical Measures for Certain Dynamical Systems with Holes, Ergodic Theory and Dynamical Systems. 30(Part 3), 687-728.

This Article is brought to you for free and open access by the Mathematics Department at DigitalCommons@Fairfield. It has been accepted for inclusion in Mathematics Faculty Publications by an authorized administrator of DigitalCommons@Fairfield. For more information, please contact digitalcommons@fairfield.edu. 


\title{
Existence and convergence properties of physical measures for certain dynamical systems with holes
}

\author{
HENK BRUIN $\dagger$, MARK DEMERS $\ddagger$ and IAN MELBOURNE $\dagger$ \\ $\dagger$ Department of Mathematics, University of Surrey, Guildford, Surrey GU2 7XH, UK \\ (e-mail: h.bruin@surrey.ac.uk,ism@math.uh.edu) \\ $\ddagger$ Department of Mathematics and Computer Science, Fairfield University, Fairfield, \\ CT 06824, USA \\ (e-mail: mdemers@fairfield.edu)
}

(Received 15 September 2008 and accepted in revised form 17 February 2009)

\begin{abstract}
We study two classes of dynamical systems with holes: expanding maps of the interval and Collet-Eckmann maps with singularities. In both cases, we prove that there is a natural absolutely continuous conditionally invariant measure $\mu$ (a.c.c.i.m.) with the physical property that strictly positive Hölder continuous functions converge to the density of $\mu$ under the renormalized dynamics of the system. In addition, we construct an invariant measure $v$, supported on the Cantor set of points that never escape from the system, that is ergodic and enjoys exponential decay of correlations for Hölder observables. We show that $v$ satisfies an equilibrium principle which implies that the escape rate formula, familiar to the thermodynamic formalism, holds outside the usual setting. In particular, it holds for Collet-Eckmann maps with holes, which are not uniformly hyperbolic and do not admit a finite Markov partition. We use a general framework of Young towers with holes and first prove results about the a.c.c.i.m. and the invariant measure on the tower. Then we show how to transfer results to the original dynamical system. This approach can be expected to generalize to other dynamical systems than the two above classes.
\end{abstract}

\section{Introduction}

Dynamical systems with holes are examples of systems whose domains are not invariant under the dynamics. Important questions in the study of such open systems include the following. What is the escape rate from the phase space with respect to a given reference measure? Starting with an initial probability measure $\mu_{0}$ and letting $\mu_{n}$ denote the distribution at time $n$ conditioned on not having escaped, does $\mu_{n}$ converge to some limiting distribution independent of $\mu_{0}$ ? Such a measure, if it exists, is a conditionally invariant measure. 
These questions have been addressed primarily for uniformly expanding or hyperbolic systems which admit finite Markov partitions: expanding maps on $\mathbb{R}^{n}$ [PY, CMS1, CMS2]; Smale horseshoes [C1, C2]; Anosov diffeomorphisms [CM1, CM2, CMT1, CMT2]; billiards with convex scatterers satisfying a non-eclipsing condition [LoM, R]; and large parameter logistic maps whose critical point maps out of the interval [HY].

Requirements on Markov partitions have been dropped for expanding maps of the interval [BaK, CV, LiM, D1]; and more recently for piecewise uniformly hyperbolic maps in two dimensions [DL]. Non-uniformly hyperbolic systems have been studied in the form of logistic maps with generic holes [D2]. Typically a restriction on the size of the hole is introduced in order to control the dynamics.

A central object of study in these open systems is the conditionally invariant measure mentioned previously. Given a self-map $\hat{T}$ of a measure space $\hat{X}$, we identify a set $H \subset \hat{X}$ which we call the hole. Once the image of a point has entered $H$, it escapes from the phase space forever. Define $X=\hat{X} \backslash H$ and $T=\left.\hat{T}\right|_{X \cap \hat{T}^{-1} X}$. A probability measure $\mu$ is called conditionally invariant if it satisfies

$$
\mu(A)=\frac{\mu\left(T^{-1} A\right)}{\mu\left(T^{-1} X\right)}
$$

for each Borel $A \subseteq X$. Iterating this relation and setting $\lambda=\mu\left(T^{-1} X\right)$, we see that $\mu\left(T^{-n} A\right)=\lambda^{n} \mu(A)$. The number $\lambda$ is called the eigenvalue of $\mu$ and $-\log \lambda$ represents its exponential rate of escape from $X$.

If $\mu$ is absolutely continuous with respect to a reference measure $m$, we call $\mu$ an absolutely continuous conditionally invariant measure and abbreviate it by a.c.c.i.m.

In [D1, D2], the author constructed Young towers to study expanding maps of the interval and unimodal Misiurewicz maps with small holes. The systems were shown to admit an a.c.c.i.m. with a density unique in a certain class of densities and converging to the Sinai-Ruelle-Bowen (SRB) measure of the closed system as the diameter of the hole tends to zero. However, left open in these papers was the question of what class of measures converges to the a.c.c.i.m. under the (renormalized) dynamics of $T$. This question is especially important for open systems since even for well-behaved hyperbolic systems, many a.c.c.i.m. may exist with overlapping supports and arbitrary escape rates [DY]. Thus it is essential to distinguish a natural a.c.c.i.m. which attracts a reasonable class of measures, including the reference measure.

The purpose of this paper is two-fold. First, we prove that for a large class of systems with holes, including:

(1) $C^{1+\alpha}$ expanding maps of the interval (see Theorem 2.10); and

(2) multimodal Collet-Eckmann maps with singularities (see Theorem 2.12),

all Hölder continuous densities $f$ which are bounded away from zero converge exponentially to the a.c.c.i.m. under the renormalized dynamics of $T$. To be precise, if $\mathcal{L}$ is the transfer operator associated with $T$ and $|\cdot|_{1}$ the $L^{1}(m)$-norm, then $\mathcal{L}^{n} f /\left|\mathcal{L}^{n} f\right|_{1}$ converges exponentially to the density of $\mu$ as $n \rightarrow \infty$. Although similar results are known for $C^{2}$ expanding maps with holes $[\mathbf{C V}, \mathbf{L i M}]$, they are completely new for multimodal maps, and even for unimodal maps without singularities. In addition, we strengthen the results on the dynamics of the tower which were used in [D1, D2]. 
Second, we study the set of non-wandering points of each system: the (measure zero) set of points, $X^{\infty}$, which never enter the hole. We construct an ergodic invariant probability measure $\tilde{v}$ supported on $X^{\infty}$ which enjoys exponential decay of correlations on Hölder functions. The measure $\tilde{v}$ is characterized by a physical limit and satisfies an equilibrium principle. This implies the generalized escape rate formula for both classes of systems in question,

$$
\log \lambda=h_{\tilde{\nu}}(T)-\int_{X} \log J T d \tilde{\nu}
$$

where $\lambda$ represents the exponential rate of escape from $X$ with respect to the reference measure $\tilde{m}, h_{\tilde{\nu}}(T)$ is the metric entropy of $T$ with respect to $\tilde{v}$, and $J T$ is the Jacobian of $T$ with respect to $\tilde{m}$.

This formula is well-known when the usual thermodynamic formalism applies (in the presence of a finite Markov partition) [Bo, C1, CM1, CMT2, CMS1]. In [BaK], an equilibrium principle was established for piecewise expanding maps with generalized potentials of bounded variation. The paper $[\mathbf{B r K}]$ deals with equilibrium states of the unbounded potential $-t \log \left|T^{\prime}\right|, t \approx 1$, for Collet-Eckmann unimodal maps $T$, using a weighted transfer operator, but not allowing any holes. Both $[\mathbf{B a K}, \mathbf{B r K}]$ use canonical Markov extensions (frequently called Hofbauer towers). In Theorem 2.17 we generalize those results to systems with holes having no Markov structure and non-uniform hyperbolicity by constructing Young towers. In contrast to previous results, we do not use bounded variation techniques and so are able to allow potentials which are piecewise Hölder continuous. This answers in the affirmative a conjecture of Chernov and van den Bedem regarding expanding maps with holes $[\mathbf{C V}]$ and a more general question raised in [DY].

Remark 1.1. It is important to note that the Young towers must be constructed for each system after the introduction of holes since the presence of holes affects return times in a possibly unbounded way. Thus existing tower constructions for the corresponding closed systems cannot be used directly.

Throughout the paper, we emphasize the physical properties of the measures involved and their characterization as push forward and pull back limits under the renormalized dynamics. In particular, the measures are independent of the Markov extensions used.

In $\S 2$, we formulate our results precisely and include a brief discussion of the issues involved. Section 3 proves the convergence results on the tower while $\S 4$ applies these results to two classes of concrete systems with holes: expanding maps of the interval and Collet-Eckmann maps with singularities. Section 5 contains proofs of the equilibrium principles for both the tower and the underlying dynamical system.

\section{Setting and statement of results}

2.1. Young towers. We recall the definition of a Young tower. Let $\hat{\Delta}_{0}$ be a measure space and let $\mathcal{Z}_{0}$ be a countable measurable partition of $\hat{\Delta}_{0}$. Given a finite reference measure $m$ on $\hat{\Delta}$, let $R$ be a function on $\hat{\Delta}_{0}$ which is constant on elements of the partition and for which $\int R d m<\infty$. We define the tower over $\hat{\Delta}_{0}$ as

$$
\hat{\Delta}=\left\{(x, n) \in \Delta_{0} \times \mathbb{N}: n<R(x)\right\},
$$


where $\mathbb{N}=\{0,1,2, \ldots\}$. We call $\hat{\Delta}_{\ell}=\left.\hat{\Delta}\right|_{n=\ell}$ the $\ell$ th level of the tower. The action of the tower map $\hat{F}$ is characterized by

$$
\begin{aligned}
\hat{F}(x, n) & =(x, n+1) \quad \text { if } n+1<R(x) \\
\hat{F}^{R(x)}(\mathcal{Z}(x)) & =\bigcup_{j \in J_{x}} Z_{j} \quad \text { for some subset of partition elements of } \mathcal{Z}_{0} \text { indexed by } J_{x}
\end{aligned}
$$

where $\mathcal{Z}_{0}(x)$ is the element of $\mathcal{Z}_{0}$ containing $x$ and $\left.\hat{F}^{R(x)}\right|_{\mathcal{Z}_{0}(x)}$ is injective.

We will abuse notation slightly and refer to a point $(x, n)$ in the tower as simply $x$ and $\hat{\Delta}_{n}$ will be made clear by the context. Also, the partition $\mathcal{Z}_{0}$ and the action of $F$ induce a natural partition of $\hat{\Delta}$ which we shall refer to by $\mathcal{Z}$, with elements $Z_{\ell, j}$ in $\hat{\Delta}_{\ell}$. With this convention, it is clear that $\mathcal{Z}$ is a Markov partition for $\hat{F}$. The definition of $R$ extends easily to the entire tower as well: $R(x)$ is simply the first time that $x$ is mapped to $\hat{\Delta}_{0}$ under $\hat{F}$. We extend $m$ to each level of the tower by setting $m(A)=m\left(\hat{F}^{-\ell} A\right)$ for every measurable set $A \subset \hat{\Delta}_{\ell}$.

2.1.1. Introduction of holes. We define a hole $H$ in $\hat{\Delta}$ as the union of countably many elements of the partition $\mathcal{Z}$, i.e., $H=\cup H_{\ell, j}$ where each $H_{\ell, j}=Z_{\ell, k}$ for some $k$. Also set $H_{\ell}=\sum_{j} H_{\ell, j}=H \cap \hat{\Delta}_{\ell}$. This preserves the Markov structure of the returns to $\hat{\Delta}_{0}$, but the definition of the return time function $R$ needs a slight modification: if $x$ is mapped into $H$ before it reaches $\hat{\Delta}_{0}, R(x)$ is defined to be the time that $x$ is mapped into $H$; otherwise, $R(x)$ remains unchanged. If $Z_{\ell, j} \subset H$, then all the elements of $\mathcal{Z}$ directly above $Z_{\ell, j}$ are deleted since once $\hat{F}$ maps a point into $H$, it disappears forever.

We will be interested in studying the dynamics of the points which have not yet fallen into the hole. To this end, we define $\Delta=\hat{\Delta} \backslash H$ and $\Delta^{n}=\bigcap_{i=0}^{n} \hat{F}^{-i} \Delta$, so $\Delta^{n}$ is the set of points which have not fallen into the hole by time $n$. Define the map $F=\left.\hat{F}\right|_{\Delta^{1}}$ and its iterates by $F^{n}=\left.\hat{F}^{n}\right|_{\Delta^{n}}$. We denote by $Z_{\ell, j}^{*} \subset \Delta$ those elements of $\mathcal{Z}$ for which $\hat{F}(Z) \subset \hat{\Delta}_{0}$. In this paper we will study the map $F$ and the transfer operator associated with it.

We consider towers with the following properties.

(P1) Exponential returns. There exist constants $C>0$ and $\theta<1$ such that $m\left(\hat{\Delta}_{n}\right) \leq C \theta^{n}$.

(P2) Generating partition. For each $x \neq y \in \Delta$, there exists a separation time $s(x, y)<$ $\infty$ such that $s(x, y)$ is the smallest non-negative integer $k$ such that $F^{k}(x)$ and $F^{k}(y)$ lie in different elements of $\mathcal{Z}$ or $\hat{F}^{k}(x), \hat{F}^{k}(y) \in H$.

(P3) Finite images. Let $\mathcal{Z}_{0}^{i m}$ be the partition of $\Delta_{0}$ generated by the sets $\left\{F^{R} Z\right\}_{Z \in \mathcal{Z}_{0}}$. We require that $\mathcal{Z}_{0}^{i m}$ be a finite partition.

Owing to (P3) we define $c_{0}:=\min _{Z^{\prime} \in \mathcal{Z}_{0}^{i m}} m\left(Z^{\prime}\right)>0$.

Using property (P2), we define a metric on $\Delta$ by $d(x, y)=\beta^{s(x, y)}$ for some $\beta \in(\theta, 1)$ where $\theta$ is as in (P1). (The value of $\beta$ may be further restricted depending on the underlying dynamical system to which we wish to apply the tower.)

We say that $(F, \Delta)$ is transitive if for each $Z_{1}^{\prime}, Z_{2}^{\prime} \in \mathcal{Z}_{0}^{i m}$, there exists an $n \in \mathbb{N}$ such that $F^{n}\left(Z_{1}^{\prime}\right) \cap Z_{2}^{\prime} \neq 0$. We say that $F$ is mixing if for each $Z^{\prime} \in \mathcal{Z}_{0}^{i m}$, there is an $N$ such that $\Delta_{0} \subset F^{n}\left(Z^{\prime}\right)$ for all $n \geq N$. 
Remark 2.1. We define mixing in this way because the usual requirement, $\operatorname{gcd}\left(\left.R\right|_{\Delta_{0}}\right)=1$, made for towers with a single base (i.e., $\mathcal{Z}_{0}^{\text {im }}$ contains a single element) is not sufficient to eliminate periodicity in towers with multiple bases.

Since we may always construct a tower with no holes in the base (by simply choosing a reference set in the underlying system which does not intersect the hole), we consider towers with no holes in $\Delta_{0}$. Define

$$
q:=\sum_{\ell \geq 1} m\left(H_{\ell}\right) \beta^{-(\ell-1)} .
$$

Our assumption on the size of the hole is

(H1) $q<(1-\beta) c_{0} /\left(1+C_{1}\right)$,

where $C_{1}$ is the distortion constant of equation (2.1) below.

Remark 2.2. If one is interested in considering towers with holes in the base, then the definition of $q$ is modified to be

$$
q:=\sum_{\ell \geq 1} m\left(H_{\ell}\right) \beta^{-(\ell-1)}+c_{0}^{-1}\left(1+C_{1}\right) m\left(H_{0}\right) \sum_{Z_{\ell, j}^{*}} m\left(Z_{\ell, j}^{*}\right) \beta^{-\ell} .
$$

Assumption (H1) remains the same and all the results of this paper apply.

2.1.2. Transfer operator. In order to study the evolution of densities according to the dynamics of $(F, \Delta)$, we introduce the transfer operator $\mathcal{L}_{F}$ defined on $L^{1}(\Delta)$ by

$$
\mathcal{L}_{F} f(x)=\sum_{y \in F^{-1} x} f(y) g(y)
$$

where $g=(d m / d(m \circ F))$. Unless otherwise noted, we will refer to $\mathcal{L}_{F}$ as simply $\mathcal{L}$ for the rest of this paper. Higher iterates of $\mathcal{L}$ are given by

$$
\mathcal{L}^{n} f(x)=\sum_{y \in F^{-n} x} f(y) g_{n}(y)
$$

where $g_{n}=g \cdot g \circ F \cdots g \circ F^{n-1}$. For $f \in L^{1}(\Delta)$, we define the Lipschitz constant of $f$ to be

$$
\operatorname{Lip}(f)=\sup _{\ell, j} \operatorname{Lip}\left(f_{\ell, j}\right) \quad \text { and } \operatorname{Lip}\left(f_{\ell, j}\right)=\sup _{x \neq y \in Z_{\ell, j}} \frac{|f(x)-f(y)|}{d(x, y)} .
$$

We will assume that $\operatorname{Lip}(\log g)<\infty$. This assumption on $g$ implies the following standard distortion estimate.

There exists a constant $C_{1}>0$ such that for every $n>0$ and for all $x, y \in \Delta$ such that $s(x, y) \geq n$, we have

$$
\left|\frac{g_{n}(x)}{g_{n}(y)}-1\right| \leq C_{1} d\left(F^{n} x, F^{n} y\right) .
$$

In particular, if $E_{n}(y)$ denotes the $n$-cylinder containing $y$ and $F^{n}\left(E_{n}(y)\right) \in \mathcal{Z}_{0}^{i m}$, then

$$
g_{n}(y) \leq\left(C_{1}+1\right) \frac{m\left(E_{n}(y)\right)}{m\left(F^{n} E_{n}(y)\right)} \leq\left(C_{1}+1\right) c_{0}^{-1} m\left(E_{n}(y)\right) .
$$


It is easy to see that $d \mu=\varphi d m$ is an a.c.c.i.m. with eigenvalue $\lambda$ if and only if $\mathcal{L} \varphi=\lambda \varphi$. Simply write for any measurable set $A \subset \Delta$,

$$
\mu\left(F^{-1} A\right)=\int_{F^{-1} A} \varphi d m=\int_{A} \mathcal{L} \varphi d m \text { and } \lambda \mu(A)=\lambda \int_{A} \varphi d m .
$$

Then the two left-hand sides are equal if and only if the two right-hand sides are equal. Thus the properties of a.c.c.i.m. for $(F, \Delta)$ are tied to the spectral properties of $\mathcal{L}$.

2.2. First results: a spectral gap for $\mathcal{L}$. We begin by proving a spectral decomposition for $\mathcal{L}$ corresponding to $(F, \Delta)$ acting on a certain Banach space of functions. The result follows essentially from Proposition 2.3 using estimates similar to those in [Y, D1]. One important difference in the present setting is that $\mathcal{L}$ does not have spectral radius 1 , as it does for systems without holes, so careful estimates are needed to ensure that a discrete spectrum exists outside the disk of radius $\beta<1$.

2.2.1. Definition of the Banach space. Let $\mathcal{V}(\Delta)$ be the set of functions on $\Delta$ which are Lipschitz continuous on elements of the partition $\mathcal{Z}$. For each $Z_{\ell, j}$ and $f \in \mathcal{V}(\Delta)$, we set $f_{\ell, j}=\left.f\right|_{Z_{\ell, j}}$. We denote by $|f|_{\infty}$ the $L^{\infty}$ norm of $f$ and define

$$
\left\|f_{\ell, j}\right\|_{\infty}:=\left|f_{\ell, j}\right|_{\infty} \beta^{\ell}, \quad\left\|f_{\ell, j}\right\|_{\operatorname{Lip}}:=\operatorname{Lip}\left(f_{\ell, j}\right) \beta^{\ell}
$$

and

$$
\|f\|=\max \left\{\|f\|_{\infty},\|f\|_{\text {Lip }}\right\}
$$

where

$$
\|f\|_{\infty}=\sup _{\ell, j}\left\|f_{\ell, j}\right\|_{\infty} \quad \text { and } \quad\|f\|_{\text {Lip }}=\sup _{\ell, j}\left\|f_{\ell, j}\right\|_{\text {Lip }} .
$$

Our Banach space is then $\mathcal{B}=\{f \in \mathcal{V}(\Delta):\|f\|<\infty\}$. The choice $\beta \in(\theta, 1)$ (where $\theta$ comes from condition (P1)) guarantees that $\mathcal{B} \subset L^{1}(\Delta)$ and the unit ball of $\mathcal{B}$ is compactly embedded in $L^{1}(\Delta)$. The proof of this fact is similar to that in [D1, Proposition 2.2].

2.2.2. Spectral picture and convergence results. Let $|\cdot|_{1}$ denote the $L^{1}$-norm with respect to $m$. In $\S 3.1$, we prove the following.

PROPOSITION 2.3. Let $(F, \Delta, H)$ be a tower with holes satisfying properties (P1)-(P3) and assumption (H1). Then there exists $C>0$ such that for each $n \in \mathbb{N}$ and all $f \in \mathcal{B}$,

$$
\left\|\mathcal{L}^{n} f\right\| \leq C \beta^{n}\|f\|_{\text {Lip }}+C|f|_{1} .
$$

Proposition 2.3, together with the compactness of the unit ball of $\mathcal{B}$ in $L^{1}(m)$ and the fact that $\left|\mathcal{L}^{n} f\right|_{1} \leq|f|_{1}$, is enough to conclude that $\mathcal{L}: \mathcal{B} \circlearrowright$ has essential spectral radius bounded by $\beta$ and spectral radius bounded by 1 [Ba]. However, since the system is open, we expect the actual spectral radius of $\mathcal{L}$ to be a constant $\lambda<1$. We must show that $\lambda>\beta$ in order to conclude that there is a spectral gap. This fact is proved in $\S 3.2$ using assumption (H1) on the measure of the hole.

Once a spectral gap has been established, the next proposition shows that the familiar spectral picture holds true for the open system. This is proved in $§ 3.2$. 
PROPOSITION 2.4. The spectral radius of $\mathcal{L}$ on $\mathcal{B}$ is $\lambda>\beta$ and $\mathcal{L}$ is quasi-compact as an operator on $\mathcal{B}$. In addition, we have the following.

(i) If $F$ is mixing, then $\lambda$ is a simple eigenvalue and all other eigenvalues have modulus strictly less than $\lambda$. Moreover, there exists $\delta>0$ such that the unique probability density $\varphi$ corresponding to $\lambda$ satisfies $\delta \lambda^{-\ell} \leq \varphi \leq \delta^{-1} \lambda^{-\ell}$ on each $\Delta_{\ell}$.

(ii) If $F$ is transitive and periodic with period $p$, then the set of eigenvalues of modulus $\lambda$ consists of simple eigenvalues $\left\{\lambda e^{2 \pi i k / p}\right\}_{k=0}^{p-1}$. The unique probability density corresponding to $\lambda$ satisfies the same bounds as in (i).

(iii) In general, $F$ has finitely many transitive components, each with its own largest eigenvalue $\lambda_{j}$. On each component, (ii) applies.

Since Proposition 2.4 eliminates the possibility of generalized eigenvectors, the projection $\Pi_{\lambda}$ onto the eigenspace of eigenvalue $\lambda$ is characterized for each $f \in \mathcal{B}$ by the limit

$$
\Pi_{\lambda} f=\lim _{n \rightarrow \infty} \frac{1}{n} \sum_{k=0}^{n-1} \lambda^{-k} \mathcal{L}^{k} f
$$

where convergence is in the $\|\cdot\|$-norm. By (iii), the eigenspace $\mathbb{V}_{\lambda}:=\Pi_{\lambda} \mathcal{B}$ has a finite basis of probability densities, each representing an a.c.c.i.m. with escape rate $-\log \lambda$.

COROLlary 2.5. Suppose that $F$ is mixing and let $\varphi \in \mathbb{V}_{\lambda}$ denote the unique probability density given by (i). Then there exists $\sigma \in(0,1)$ and $C \geq 1$ such that

$$
\left\|\lambda^{-n} \mathcal{L}^{n} f-c(f) \varphi\right\| \leq C\|f\| \sigma^{n},
$$

for all $f \in \mathcal{B}$ where $c(f)$ is a constant depending on $f$.

Proof. The operator $\lambda^{-1} \mathcal{L}: \mathcal{B} \rightarrow \mathcal{B}$ has spectral radius 1 and essential spectral radius $\beta \lambda^{-1}$. Moreover, there is a simple eigenvalue at 1 with eigenspace $\mathbb{V}_{\lambda}$ spanned by $\varphi$ and no further eigenvalues on the unit circle. Hence there is an $\mathcal{L}$-invariant closed splitting $\mathcal{B}=\mathbb{V}_{\lambda} \oplus \mathbb{W}_{\lambda}$ and $\mathcal{L}: \mathbb{W}_{\lambda} \rightarrow \mathbb{W}_{\lambda}$ has spectral radius $\rho \in\left(\beta \lambda^{-1}, 1\right)$. The result follows for any $\sigma \in(\rho, 1)$.

The expression in Corollary 2.5 is not satisfactory, however, if one wishes to obtain an approximation of the conditionally invariant measure when the eigenvalue is not known in advance. In such a case, the object of interest is the limit $\mathcal{L}^{n} f /\left|\mathcal{L}^{n} f\right|_{1}$ as $n \rightarrow \infty$ where the density is renormalized at each step.

Proposition 2.6. Let $(F, \Delta)$ be mixing and satisfy the hypotheses of Proposition 2.3, and let $f \in \mathcal{B}$. Then $c(f)>0$ if and only if

$$
\lim _{n \rightarrow \infty} \frac{\mathcal{L}^{n} f}{\left|\mathcal{L}^{n} f\right|_{1}}=\varphi
$$

where convergence is in the $\|\cdot\|$-norm. Moreover convergence is at the rate $\sigma^{n}$ where $\sigma$ is as in Corollary 2.5.

Proof. Note that $\lambda^{-n}\left|\mathcal{L}^{n} f\right|_{1} \rightarrow|c(f)|$ by Corollary 2.5 so that if $c(f)>0$, we may write

$$
\lim _{n \rightarrow \infty} \frac{\mathcal{L}^{n} f}{\left|\mathcal{L}^{n} f\right|_{1}}=\lim _{n \rightarrow \infty} \frac{\mathcal{L}^{n} f}{\lambda^{n}} \frac{\lambda^{n}}{\left|\mathcal{L}^{n} f\right|_{1}}=\varphi .
$$


The converse follows from the linear structure of $\mathcal{L}$. We write $\mathcal{B}=\mathbb{V}_{\lambda} \oplus \mathbb{W}_{\lambda}$ as in the proof of Corollary 2.5. Then $\mathbb{W}_{\lambda}=\{g \in \mathcal{B}: c(g)=0\}$.

Remark 2.7. In what follows, we will be interested in establishing which functions satisfy $c(f)>0$, first on the tower and then for the concrete systems for which towers are constructed. Proposition 3.3 guarantees that in particular $c(1)>0$ so that the reference measure on $\Delta$ converges to the a.c.c.i.m.

2.3. An equilibrium principle for $(F, \Delta)$. The characterization of $\varphi$ in terms of the physical limit $\mathcal{L}^{n} f /\left|\mathcal{L}^{n} f\right|_{1}$ allows us to construct an invariant measure $v$ singular with respect to $m$ and supported on $\Delta^{\infty}=\bigcap_{n=0}^{\infty} \Delta^{n}$, the set of points which never enter the hole. Although $v$ is supported on a zero $m$-measure Cantor-like set, the results of this section indicate that it is physically relevant to the system.

To state our results, we first introduce a new Banach space $\mathcal{B}_{0}$ consisting of functions that are uniformly bounded and uniformly locally Lipschitz. More precisely, let $|f|_{\infty}$ denote the standard sup-norm. Then define $|f|_{\text {Lip }}=\sup _{\ell, j} \operatorname{Lip}\left(f_{\ell, j}\right)$ and $\|f\|_{0}=$ $\max \left\{|f|_{\infty},|f|_{\text {Lip }}\right\}$. Note that contrary to $\|f\|_{\text {Lip }}$, the seminorm $|f|_{\text {Lip }}$ does not have the weights $\beta^{\ell}$. Finally, let

$$
\mathcal{B}_{0}:=\left\{f \in \mathcal{B}:\|f\|_{0}<\infty\right\} .
$$

The following proposition is proved in $\S 3.3$.

Proposition 2.8. Suppose $(F, \Delta)$ satisfies properties $(P 1)-(P 3)$ and $(H 1)$ of $\$ 2.1 .1$ and is mixing. Then $(F, \Delta)$ admits an invariant probability measure $v$ supported on $\Delta^{\infty}$, which satisfies

$$
v(f)=\lim _{n \rightarrow \infty} \lambda^{-n} \int_{\Delta^{n}} f d \mu
$$

for all $f \in \mathcal{B}_{0}$. In addition, $v$ is ergodic and

$$
\left|\int_{\Delta^{\infty}} f_{1} f_{2} \circ F^{n} d v-v\left(f_{1}\right) v\left(f_{2}\right)\right| \leq C\left\|f_{1}\right\|\left|f_{2}\right|_{\infty} \sigma^{n}
$$

for all $f_{1}, f_{2} \in \mathcal{B}_{0}, n \geq 1$.

In addition to its characterization as a limit, the invariant measure $v$ is natural to the system in the sense that it satisfies the below equilibrium principle.

Let

$$
v_{0}:=\left.\frac{1}{v\left(\Delta_{0}\right)} v\right|_{\Delta_{0}}
$$

and note that $\nu_{0}$ is an invariant measure for $F^{R}$ on $\Delta^{\infty} \cap \Delta_{0}$. Proposition 5.1 shows that in fact $v_{0}$ is a Gibbs measure for $F^{R}$.

We call a measure $\eta$ non-singular provided $\eta(F(A))=0$ if and only if $\eta(A)=0$. The following theorem is proved in $\$ 5$.

Theorem 2.9. Let $(F, \Delta)$ satisfy the hypotheses of Proposition 2.8. Let $\mathcal{M}_{F}$ be the set of $F$-invariant Borel probability measures on $\Delta$. Then

$$
\log \lambda=\sup _{\eta \in \mathcal{M}_{F}}\left\{h_{\eta}(F)-\int_{\Delta} \log J F d \eta\right\}
$$


where $h_{\eta}(F)$ is the metric entropy of $\eta$ with respect to $F$ and $J F$ is the Jacobian of $F$ with respect to $m$. In addition, $v$ is the unique non-singular measure in $\mathcal{M}_{F}$ which attains the supremum.

2.4. Applications to specific dynamical systems. We apply the results about abstract towers with holes to two specific classes of dynamical systems with holes: $C^{1+\alpha}$ piecewise expanding maps of the interval and locally $C^{2}$ multimodal Collet-Eckmann maps with singularities.

2.4.1. Piecewise expanding maps of the interval. By a piecewise expanding map of the unit interval $\hat{I}$, we mean a map $\hat{T}: \hat{I} \circlearrowleft$ satisfying the following properties. There exists a partition of $\hat{I}$ into finitely many intervals, $\hat{I}_{j}$, such that (a) $\hat{T}$ is $C^{1+\alpha}$ and monotonic on each $\hat{I}_{j}$ for some $\alpha>0$; and (b) $\left|\hat{T}^{\prime}\right| \geq \tau>2$. Note that we can always satisfy (b) if $\left|\hat{T}^{\prime}\right| \geq 1+\varepsilon$ by considering a higher iterate of $\hat{T}$.

Let $\hat{I}_{j}^{n}$ denote the intervals of monotonicity for $\hat{T}^{n}$. The uniform expansion of $\hat{T}$ implies the following familiar distortion bound: there exists a constant $C_{3}>0$ such that for any $n$, if $x$ and $y$ belong to the same $\hat{I}_{j}^{n}$, then

$$
\left|\frac{\left(\hat{T}^{n}\right)^{\prime}(x)}{\left(\hat{T}^{n}\right)^{\prime}(y)}-1\right| \leq C_{3}\left|\hat{T}^{n}(x)-\hat{T}^{n}(y)\right|^{\alpha} .
$$

Introduction of holes. A hole $\tilde{H}$ in $[0,1]$ is a finite union of open intervals $\tilde{H}_{j}$. (We use the ${ }^{\sim}$ to distinguish from the hole on the tower.) Let $I=\hat{I} \backslash \tilde{H}$ and for $n \geq 0$, define $I^{n}=\bigcap_{i=0}^{n} \hat{T}^{-i} I$. We are interested in studying the dynamics of $T^{n}:=\left.\hat{T}^{n}\right|_{I^{n}}$.

Let $\gamma$ be the length of the shortest interval of monotonicity of $T$. Our sole condition on the hole is

(H2) $\tilde{m}(\tilde{H}) \leq \gamma^{2}(1-\beta)\left(\tau-2 \beta^{-1}\right) /\left(1+C_{3}\right)$

where $\tilde{m}$ is Lebesgue measure on $\hat{I}$ and $\beta>\max \left\{2 \tau^{-1}, \tau^{-\alpha}\right\}$.

The following theorem is proved in [D1].

THEOREM A. [D1] Let $T$ be a $C^{1+\alpha}$ piecewise expanding map of the interval and let $\tilde{H}$ be a hole satisfying the bound given in $(H 2)$. Then $(T, I)$ admits a tower $(F, \Delta)$ which satisfies properties $(P 1)-(P 3)$ and $(H 1)$ of $\$ 2.1 .1$ as well as $(A 1)$ of $\$ 4.1$ with $\theta=2 / \tau$, $C_{1}=C_{3}, c_{0}=\gamma$ and $C_{2}=1$.

If in addition, a transitivity condition is satisfied, then there is a unique conditionally invariant density $\varphi \in \mathcal{B}$ with eigenvalue $\lambda$.

In order to eliminate periodicity and ensure transitivity for the map $T$ and for the tower, we can impose the following transitivity condition.

(T1) Let $J$ be an interval of monotonicity for $T$. There exists an $n_{1}>0$ such that $T^{n_{1}} J$ covers $I$ up to finitely many points.

Property (T1) is analogous to the covering property for piecewise expanding maps of the interval without holes which is a necessary and sufficient condition for the existence of a unique absolutely continuous invariant measure whose density is bounded away from zero (see [Li]). 
Fix $\bar{\alpha} \geq-\log \beta / \log \tau$ and if necessary, choose $\beta$ closer to 1 so that $\bar{\alpha} \leq \alpha$. Let $I^{\infty}$ denote the set of points which never escape from $I$ and define

$$
\mathcal{G}=\left\{\tilde{f} \in C^{\bar{\alpha}}(I): \tilde{f}>0 \text { on } I^{\infty}\right\} .
$$

Denote by $\mathcal{L}_{T}$ the transfer operator of $T$ with respect to $\tilde{m}$ and let $|\cdot|_{1}$ denote the $L^{1}(\tilde{m})$ norm. We prove the following theorem in $\$ 4.2$.

THEOREM 2.10. Let $T$ satisfy the hypotheses of Theorem A in addition to condition (T1).

There exists $\lambda>0$ such that for all $\tilde{f} \in \mathcal{G}$, the escape rate with respect to $\tilde{\eta}=\tilde{f} \tilde{m}$ is well-defined and equal to $-\log \lambda$, i.e.,

$$
\lim _{n \rightarrow \infty} \frac{1}{n} \log \tilde{\eta}\left(I^{n}\right)=\log \lambda .
$$

There exists a unique a.c.c.i.m. $\tilde{\mu}$ with density $\tilde{\varphi}$ and eigenvalue $\lambda$ such that for all $\tilde{f} \in \mathcal{G}$, we have

$$
\left|\frac{\mathcal{L}_{T}^{n} \tilde{f}}{\left|\mathcal{L}_{T}^{n} \tilde{f}\right|_{1}}-\tilde{\varphi}\right|_{1} \leq C_{T}|\tilde{f}|_{C^{\bar{\alpha}}} \sigma^{n}
$$

for some $\sigma \in(\beta, 1)$ and $C_{T}$ depends only on the smoothness and distortion of the map $T$.

Remark 2.11. The results of Theorems A and 2.10 generalize those obtained in $[\mathbf{C V}, \mathbf{L i M}]$ for $C^{2}$ expanding maps using bounded variation techniques. We make no assumptions on the position of the holes, only their measure.

2.4.2. Collet-Eckmann maps with singularities. Collet-Eckmann maps are interval maps with critical points such that the derivatives $D \hat{T}^{n}$ at the critical values increase exponentially. We will follow the approach of [DHL] which allows for discontinuities and points with infinite derivative as in Lorenz maps. (We try to use the same notation as in [DHL], but adding a $*$ if there is a clash with our own notation.) The map $\hat{T}: \hat{I} \rightarrow \hat{I}$ is locally $C^{2}$ and has a critical set Crit $=\mathrm{Crit}_{c} \cup \mathrm{Crit}_{s}$ consisting respectively of genuine critical points $c$ with critical order $1<\ell_{c}<\infty$ and singularities with critical order $0<\ell_{c} \leq 1$. At each of these points $\hat{T}$ is allowed to have a discontinuity as well, so $c \in$ Crit has a left and right critical order which need not be the same. Furthermore, $\hat{T}$ satisfies the following conditions for all $\delta>0$ (where $B_{\delta}\left(\right.$ Crit) $=\bigcup_{c \in \text { Crit }} B_{\delta}(c)$ is a $\delta$-neighborhood of Crit).

(C1) Expansion outside $B_{\delta}$ (Crit). There exist $\lambda^{*}>0$ and $\kappa>0$ such that for every $x$ and $n \geq 1$ such that $x_{0}=x, \ldots, x_{n-1}=\hat{T}^{n-1}(x) \notin B_{\delta}($ Crit $)$, we have $\dagger$

$$
\left|D \hat{T}^{n}(x)\right| \geq \kappa \delta^{\ell_{\max }-1} e^{\lambda^{*} n},
$$

where $\ell_{\max }=\max \left\{\ell_{c}: c \in \mathrm{Crit}_{c}\right\}$. Moreover, if $x_{0} \in \hat{T}\left(B_{\delta}(\mathrm{Crit})\right)$ or $x_{n} \in B_{\delta}(\mathrm{Crit})$, then we have

$$
\left|D \hat{T}^{n}(x)\right| \geq \kappa e^{\lambda^{*} n} .
$$

$\dagger$ The addition of exponent $\ell_{\max }-1$ for $\delta$ in this formula is a correction to [DHL, Condition (H1)], which affects the proofs only in the sense that some constants will be different. The formula in [DHL] cannot be realized for any $x$ at distance $\delta$ to any critical point $c \in \mathrm{Crit}_{s}$ with $\ell_{c}>2$. 
(C2) Slow recurrence and derivative growth along critical orbit. There exists $\Lambda^{*}>0$ such that for all $c \in \mathrm{Crit}_{c}$ there is $\alpha_{c}^{*} \in\left(0, \Lambda^{*} /\left(5 \ell_{c}\right)\right)$ such that $\dagger$

$$
\left|D \hat{T}^{k}(\hat{T}(c))\right| \geq e^{\Lambda^{*} k} \quad \text { and } \quad \operatorname{dist}\left(\hat{T}^{k}(c), \text { Crit }\right)>\delta e^{-\alpha_{c}^{*} k} \quad \text { for all } k \geq 1 .
$$

(C3) Density of preimages. There exists $c^{*} \in$ Crit whose preimages are dense in $\hat{I}$, and no other critical point is among these preimages.

Condition (C1) follows for piecewise $C^{2}$ maps from Mañé's theorem, see [MS, Ch. III.5]. The first half of condition (C2) is the actual Collet-Eckmann condition, and the second half is a slow recurrence condition. Condition (C3) excludes the existence of non-repelling periodic points.

In [DHL], $\alpha_{c}^{*}$ is assumed to be small relative to $\lambda^{*}$ and $\Lambda^{*}$. We keep the same restriction on $\alpha_{c}^{*}$ and do not need to shrink it further after the introduction of holes.

Introduction of holes. In order to apply the tower construction of [DHL] to our setting, we place several conditions on the placement of the holes in the interval $\hat{I}$. We adopt notation similar to that in $\$ 2.4 .1$.

A hole $\tilde{H}$ in $\hat{I}$ is a finite union of open intervals $\tilde{H}_{j}, j=1, \ldots, L$. Let $I=\hat{I} \backslash \tilde{H}$ and set $I^{n}=\bigcap_{i=0}^{n} \hat{T}^{-i} I$. Define $T=\hat{T} \mid I^{1}$ and let $\tilde{m}$ denote Lebesgue measure on $\hat{I}$.

(B1) Let $\alpha_{c}^{*}>0$ be as in (C2). For all $c \in \mathrm{Crit}_{c}$ and $k \geq 0$,

$$
\operatorname{dist}\left(\hat{T}^{k}(c), \partial \tilde{H}\right)>\delta e^{-\alpha_{c}^{*} k} .
$$

Our second condition on $\tilde{H}$ is that the positions of its connected components are generic with respect to one another. This condition will also double as a transitivity condition on the constructed tower which ensures our conditionally invariant density will be bounded away from zero. In order to formulate this condition, we need the following fact about $C^{2}$ non-flat non-renormalizable maps satisfying $(\mathrm{C} 1)-(\mathrm{C} 2)$.

For all $\delta>0$ there exists $n=n(\delta)$ such that for all intervals $\omega \subseteq \hat{I}$ with $|\omega| \geq \delta / 3$ :

(i) $\hat{T}^{n} \omega \supseteq \hat{I}$; and

(ii) there is a subinterval $\omega^{\prime} \subset \omega$ such that $\hat{T}^{n^{\prime}}$ maps $\omega^{\prime}$ diffeomorphically onto $\left(c^{*}-3 \delta, c^{*}+3 \delta\right)$ for some $0<n^{\prime} \leq n$.

We also need some genericity conditions on the placement of the components of the hole. Within each component $\tilde{H}_{j}$, we place an artificial critical point $b_{j}$, so Crit $_{\text {hole }}=$ $\left\{b_{1}, \ldots, b_{L}\right\}$. The points $b_{j}$ are positioned so that the following holds.

(B2) (a) $\operatorname{orb}\left(b_{j}\right) \cap c=\emptyset$ for all $1 \leq j \leq L$ and $c \in$ Crithole $\cup$ Crit $_{c}$.

(b) Let $\hat{T}^{-1}\left(\hat{T} b_{j}\right)=\bigcup_{i=1}^{K_{j}} g_{j, i}$. For all $j, k \in\{1, \ldots, L\}$, there exists $i \in\{1, \ldots$, $\left.K_{j}\right\}$ such that $\hat{T}^{\ell} b_{k} \neq g_{j, i}$ for $1 \leq \ell \leq n(\delta)$.

Here $n(\delta)$ is the integer corresponding to $\delta$ in (2.4) and $\delta$ is chosen so small that: (i) all points in $\mathrm{Crit}_{c} \cup \mathrm{Crit}_{s} \cup \mathrm{Crit}_{\text {hole }}$ are at least $2 \delta$ apart; and (ii) for each $j=1, \ldots, L$, there is $\tau=\tau(j) \geq 1$ such that

$$
\left|D \hat{T}^{\tau}(x)\right| \geq \max \left\{\kappa e^{\lambda^{*} \tau}, 6\right\} \quad \text { for all } x \in B_{\delta}\left(b_{j}\right) .
$$

$\dagger$ The fact that $\alpha_{c}^{*}$ depends on $c$ in this way is a correction to [DHL, Condition (H2)], where this is not stated, but used in the [DHL, proof of Lemma 2]. 
Condition (C1) implies that $\left|D \hat{T}^{\tau}(x)\right| \geq \kappa e^{\lambda^{*} \tau}$ whenever $x \notin B_{\delta}\left(\mathrm{Crit}_{c}\right)$ and $T^{\tau}(x) \in$ $B_{\delta}\left(\mathrm{Crit}_{c}\right)$, so by taking $\delta$ small, and using assumption (B2)(a), we can indeed find $\tau$ such that also $\left|D \hat{T}^{\tau}(x)\right| \geq 6$.

As before, let $I^{\infty}$ denote the set of points which never escapes from $I$ and let $\mathcal{G}=\{\tilde{f} \in$ $C^{\bar{\alpha}}(I): \tilde{f}>0$ on $\left.I^{\infty}\right\}$. We prove the following theorem in $\S 4.3$.

THEOREM 2.12. Let $\hat{T}$ be a non-renormalizable map satisfying conditions $(C 1)-(C 3)$ and let $\tilde{H}$ be a sufficiently small hole satisfying (B1)-(B2).

There exists $\lambda>0$ such that for all $\tilde{f} \in \mathcal{G}$, the escape rate with respect to $\tilde{\eta}=\tilde{f} \tilde{m}$ is well-defined and equal to $-\log \lambda$, i.e.,

$$
\lim _{n \rightarrow \infty} \frac{1}{n} \log \tilde{\eta}\left(I^{n}\right)=\log \lambda
$$

Moreover, there exists a unique a.c.c.i.m. $\tilde{\mu}$ with density $\tilde{\varphi}$ and eigenvalue $\lambda$ such that for all $\tilde{f} \in \mathcal{G}$, we have

$$
\left|\frac{\mathcal{L}_{T}^{n} \tilde{f}}{\left|\mathcal{L}_{T}^{n} \tilde{f}\right|_{1}}-\tilde{\varphi}\right|_{1} \leq C_{T}|\tilde{f}|_{C^{\bar{\alpha}}} \tilde{\sigma}^{n}
$$

for some $\tilde{\sigma} \in(\beta, 1)$ and $C_{T}$ depends only on the smoothness and distortion of the map $T$.

Remark 2.13. When $\hat{T}$ is a Misiurewicz map (i.e., all critical points are non-recurrent and all periodic points are non-repelling), it is possible to give a constructive bound on the size of the hole in terms of explicit constants. In this case we assume that the critical point does not fall into the hole, but there is no need for condition (B2)(a), see [D2, §2.2].

Small hole limit. Fix $L$ distinct points $b_{1}, \ldots, b_{L} \in \hat{I}$ which we consider to be infinitesimal holes satisfying (B1) and (B2). We call this hole of measure zero $\tilde{H}^{(0)}$ with components $\tilde{H}_{j}^{(0)}=b_{j}, j=1, \ldots, L$. For each $h>0$, we then define a family of holes $\mathcal{H}(h)$ such that $\tilde{H} \in \mathcal{H}(h)$ if and only if the following hold.

(1) $b_{j} \in \tilde{H}_{j}$ and $\tilde{m}\left(\tilde{H}_{j}\right) \leq h$ for each $1 \leq j \leq L$.

(2) $\tilde{H}$ satisfies (B1).

When we shrink a hole in $\mathcal{H}(h)$, we keep $b_{1}, \ldots, b_{L}$ fixed and simply choose a smaller $h$. The following theorem is proved in $\$ 4.3 .1$.

THEOREM 2.14. Let $\hat{T}$ satisfy the hypotheses of Theorem 2.12 and let $\tilde{H}^{(h)} \in \mathcal{H}(h)$ be a family of holes. Let $d \tilde{\mu}_{h}=\tilde{\varphi}_{h} d \tilde{m}$ be the a.c.c.i.m. given by Theorem 2.12 with eigenvalue $\lambda_{h}$. Then $\lambda_{h} \rightarrow 1$ and $\tilde{\mu}_{h}$ converges weakly to the unique SRB measure for $\hat{T}$ as $h \rightarrow 0$.

Remark 2.15. A similar theorem was proved for piecewise expanding maps in [D1] and for Misiurewicz maps in [D2].

2.5. An equilibrium principle for $(T, X)$. In this section, $T$ is either an expanding map satisfying the assumptions of Theorem 2.10 or a Collet-Eckmann map with singularities satisfying the assumptions of Theorem 2.12.

Recall the invariant measure $v$ supported on $\Delta^{\infty}$ introduced in Proposition 2.8. The measure $\tilde{v}:=\pi_{*} v$ is $T$-invariant and is supported on $X^{\infty}=\pi\left(\Delta^{\infty}\right)$. We show that $\tilde{v}$ is physically relevant to the system $(T, X)$ in two ways. 
THEOREM 2.16. The invariant measure $\tilde{v}$ is characterized by

$$
\tilde{v}(\tilde{f})=\lim _{n \rightarrow \infty} \lambda^{-n} \int_{X^{n}} \tilde{f} d \tilde{\mu}
$$

for all functions $\tilde{f} \in C^{\bar{\alpha}}(X)$. In addition, $\tilde{v}$ is ergodic and enjoys exponential decay of correlations on Hölder observables.

Although $\tilde{v}$ is defined simply as $\pi_{*} \nu$, the preceding theorem gives a characterization of $\tilde{v}$ which is independent of the tower construction. This is important for two reasons: first, it implies that two different tower constructions will yield the same invariant measure; second, it eliminates the need to construct a tower in order to compute $\tilde{v}$.

The second theorem is a consequence of Theorem 2.9.

TheOREM 2.17. Let $\mathcal{M}_{T}^{\prime}=\pi_{*} \mathcal{M}_{F}=\left\{\pi_{*} \eta: \eta \in \mathcal{M}_{F}\right\}$ be the set of T-invariant Borel probability measures on $X$ whose lift to $\Delta$ is well-defined. Then

$$
\log \lambda=\sup _{\eta \in \mathcal{M}_{T}^{\prime}}\left\{h_{\eta}(T)-\int_{X} \log J T d \eta\right\}
$$

where $J T$ is the Jacobian of $T$ with respect to $\tilde{m}$. The invariant measure $\tilde{v}$ is the unique non-singular measure $\tilde{\eta}$ in $\mathcal{M}_{T}^{\prime}$ which attains the supremum.

Theorem 2.16 is proved in $\$ 5.1$ and Theorem 2.17 is proved in $\S 5.3$.

\section{Convergence properties of $\mathcal{L}$}

3.1. Lasota-Yorke inequalities. In this section we prove Proposition 2.3 by deriving Lasota-Yorke type inequalities for $\|\cdot\|_{\infty}$ and $\|\cdot\|_{\text {Lip }}$,

$$
\left\|\mathcal{L}^{n} f\right\|_{\infty} \leq C \beta^{n}\|f\|_{\text {Lip }}+C|f|_{1}, \quad\left\|\mathcal{L}^{n} f\right\|_{\text {Lip }} \leq C \beta^{n}\|f\|_{\text {Lip }}+C|f|_{1} .
$$

Proof of Proposition 2.3. We fix $n \in \mathbb{N}$ and separate the estimates into two parts: those for $Z_{\ell, j}$ with $\ell \geq n$ and those with $\ell<n$.

Estimate \# 1. For any $x \in Z_{\ell, j}$ with $\ell \geq n$ and $f \in \mathcal{B}$, note that $\mathcal{L}^{n} f(x)=f\left(F^{-n} x\right)$ since $g_{n}\left(F^{-n} x\right)=1$. This allows us to estimate

$$
\left\|\mathcal{L}^{n} f_{\ell, j}\right\|_{\infty}:=\left|f\left(F^{-n}\right)_{\ell, j}\right|_{\infty} \beta^{\ell}=\left(\left|f_{\ell-n, j}\right|_{\infty} \beta^{\ell-n}\right) \beta^{n}=\left\|f_{\ell-n, j}\right\|_{\infty} \beta^{n} .
$$

Estimate \#2. Again choose any $Z_{\ell, j}$ with $\ell \geq n$. Then

$$
\begin{aligned}
\left\|\mathcal{L}^{n} f_{\ell, j}\right\|_{\text {Lip }} & :=\sup _{x, y \in Z_{\ell, j}} \frac{\left|f\left(F^{-n} x\right)-f\left(F^{-n} y\right)\right|}{d(x, y)} \beta^{\ell} \\
& =\beta^{n} \sup _{x, y \in Z_{\ell, j}} \frac{\left|f\left(F^{-n} x\right)-f\left(F^{-n} y\right)\right|}{d\left(F^{-n} x, F^{-n} y\right) \beta^{-n}} \beta^{\ell-n}=\beta^{2 n}\left\|f_{\ell-n, j}\right\|_{\text {Lip }}
\end{aligned}
$$

since $s(x, y)=s\left(F^{-n} x, F^{-n} y\right)-n$. 
Estimate \#3. Let $x \in Z_{0, j}$ be a point in $\Delta_{0}$. We denote by $\mathcal{E}_{n}$ the connected components of $F^{-n}\left(Z^{\prime}\right)$ for $Z^{\prime} \in \mathcal{Z}_{0}^{i m}$ and let $E_{n}(y)$ denote the element of $\mathcal{E}_{n}$ containing $y$.

$$
\left|\mathcal{L}^{n} f(x)\right| \leq \sum_{y \in F^{-n} x}|f(y)| g_{n}(y) \leq \sum_{y \in F^{-n} x}|f(\bar{a})| g_{n}(y)+g_{n}(y) \frac{|f(y)-f(\bar{a})|}{d(y, \bar{a})} d(y, \bar{a})
$$

where $\bar{a} \in E_{n}(y)$ is any point satisfying

By (2.2),

$$
|f(\bar{a})| \leq \frac{1}{m\left(E_{n}(y)\right)} \int_{E_{n}(y)}|f| d m .
$$

$$
g_{n}(y) \leq\left(C_{1}+1\right) c_{0}^{-1} m\left(E_{n}(y)\right) .
$$

Finally, note that $F^{n} y, F^{n} \bar{a} \in Z_{0, j}$ so that $n$ is a return time for $y$ and $\bar{a}$. If $y \in \Delta_{\ell(y)}$, then the definition of $d$ from $\S 2.1 .1$ implies $d(y, \bar{a}) \leq \beta^{n}$.

Putting this together with (3.1), we estimate

$$
\begin{aligned}
\left|\mathcal{L}^{n} f(x)\right| & \leq \sum_{y \in F^{-n} x} \frac{g_{n}(y)}{m\left(E_{n}(y)\right)} \int_{E_{n}(y)}|f| d m+g_{n}(y) \beta^{-\ell(y)}\|f\|_{\text {Lip }} \beta^{n} \\
& \leq \sum_{y \in F^{-n} x}\left(1+C_{1}\right) c_{0}^{-1} \int_{E_{n}(y)}|f| d m+\left(1+C_{1}\right) c_{0}^{-1} m\left(E_{n}(y)\right) \beta^{n-\ell(y)}\|f\|_{\text {Lip }} \\
& \leq\left(1+C_{1}\right) c_{0}^{-1} \int_{\Delta^{n}}|f| d m+C \beta^{n}\|f\|_{\text {Lip }}
\end{aligned}
$$

where the second sum is finite since $\beta>\theta$.

Estimate \#4. Let $x, y \in Z_{0, j}$, and let $x^{\prime} \in F^{-n} x, y^{\prime} \in F^{-n} y$, denote preimages taken along the same branch of $F^{-n}$. Then summing over all inverse branches gives

$$
\begin{aligned}
\frac{\left|\mathcal{L}^{n} f(x)-\mathcal{L}^{n} f(y)\right|}{d(x, y)} & \leq \sum_{x^{\prime} \in F^{-n} x} \frac{\left|g_{n}\left(x^{\prime}\right) f\left(x^{\prime}\right)-g_{n}\left(y^{\prime}\right) f\left(y^{\prime}\right)\right|}{d(x, y)} \\
& \leq \sum_{x^{\prime} \in F^{-n} x} g_{n}\left(x^{\prime}\right) \frac{\left|f\left(x^{\prime}\right)-f\left(y^{\prime}\right)\right|}{d(x, y)}+\left|f\left(y^{\prime}\right)\right| \frac{\left|g_{n}\left(x^{\prime}\right)-g_{n}\left(y^{\prime}\right)\right|}{d(x, y)} \\
& \leq \sum_{x^{\prime} \in F^{-n} x}\left(1+C_{1}\right) c_{0}^{-1} m\left(E_{n}\left(x^{\prime}\right)\right)\|f\|_{\text {Lip }} \beta^{n-\ell\left(x^{\prime}\right)}+\left|f\left(y^{\prime}\right)\right| C_{1} g_{n}\left(y^{\prime}\right)
\end{aligned}
$$

where we have used (2.1) and (3.1) for $g_{n}$ in the last line as well as the fact that $d\left(x^{\prime}, y^{\prime}\right)=\beta^{n} d(x, y)$ for $x^{\prime} \in \Delta_{\ell\left(x^{\prime}\right)}$. The second sum is identical to that in Estimate \#3. Thus

$$
\frac{\left|\mathcal{L}^{n} f(x)-\mathcal{L}^{n} f(y)\right|}{d(x, y)} \leq C \beta^{n}\|f\|_{\text {Lip }}+C|f|_{1} .
$$

Now on $Z_{\ell, j}$ with $\ell<n$, we can combine Estimates \#1 and \#3 to obtain

$$
\left\|\mathcal{L}^{n} f_{\ell, j}\right\|_{\infty} \leq \beta^{\ell}\left\|\mathcal{L}^{n-\ell} f_{0, j}\right\|_{\infty} \leq \beta^{\ell}\left(C \beta^{n-\ell}\|f\|_{\text {Lip }}+C|f|_{1}\right)
$$

which implies the estimate for the $\|\cdot\|_{\infty}$-norm.

Similarly, we can combine Estimates \#2 and \#4 to obtain

$$
\left\|\mathcal{L}^{n} f_{\ell, j}\right\|_{\text {Lip }} \leq \beta^{2 \ell}\left\|\mathcal{L}^{n-\ell} f_{0, j}\right\|_{\text {Lip }} \leq \beta^{2 \ell}\left(C \beta^{n-\ell}\|f\|_{\text {Lip }}+C|f|_{1}\right)
$$

which completes the estimate for the $\|\cdot\|_{\text {Lip }}$-norm. 
3.2. Spectral gap. Although Proposition 2.3 implies that the essential spectral radius of $\mathcal{L}$ on $\mathcal{B}$ is less than or equal to $\beta$, we must still ensure that there is a spectral gap, i.e., that there is an eigenvalue $\beta<\lambda<1$ which represents the rate of escape of typical elements of $\mathcal{B}$.

This fact follows from the bound on the measure of the hole $H$ given by (H1). To prove it, it will be convenient to recall some results from [D1] which concern the nonlinear operator $\mathcal{L}_{1} f:=\mathcal{L} f /|\mathcal{L} f|_{1}$. Thus $\mathcal{L}_{1} f$ represents the normalized push-forward density which is conditioned on non-absorption by the hole. In [D1] it was shown that for small holes, $\mathcal{L}_{1}$ preserves a convex subset of $\mathcal{B}$ defined by

$$
\mathcal{B}_{M}=\left\{f \in \mathcal{B}: f \geq 0,|f|_{1}=1,\|f\|_{\infty} \leq M,\|f\|_{\log } \leq M\right\}
$$

where

$$
\|f\|_{\log }=\sup _{\ell, j} \operatorname{Lip}\left(\log f_{\ell, j}\right) .
$$

We include the proof of the proposition here for clarity and also to formulate the bound on $H$ in terms of the present notation.

Proposition 3.1. Let $M \in\left(\left(1+C_{1}\right) c_{0}^{-1},(1-\beta) q^{-1}\right)$. Then the following hold.

(i) $\mathcal{L}_{1}^{n}$ maps $\mathcal{B}_{M}$ into itselffor $n$ sufficiently large.

(ii) There exists $\beta^{\prime}>\beta$ such that $|\mathcal{L} f|_{1} \geq \beta^{\prime}$ for all $f \in \mathcal{B}_{M}$.

Proof. Note that $\operatorname{Lip}\left(\log f_{\ell, j}\right)$ is equivalent to

$$
\sup _{x, y \in Z_{\ell, j}} \frac{|f(x)-f(y)|}{f(x) d(x, y)} .
$$

We will work with this expression in the following estimates. For $f \in \mathcal{B}_{M}$, we prove the analogue of Estimates \#1-\#4 from $\$ 3.1$ using $\|\cdot\|_{\log }$. Estimates \#1 and \#2 are the same so we do not repeat those.

To prove Estimate \#3, note that equation (3.2) becomes

$$
\begin{aligned}
\left|\mathcal{L}^{n} f(x)\right| & \leq \sum_{y \in F^{-n} x} f(\bar{a}) g_{n}(y)+g_{n}(y) \frac{|f(y)-f(\bar{a})|}{f(\bar{a}) d(y, \bar{a})} d(y, \bar{a}) f(\bar{a}) \\
& \leq \sum_{y \in F^{-n} x} \frac{g_{n}(y)}{m\left(E_{n}(y)\right)} \int_{E_{n}(y)} f d m+\frac{g_{n}(y)}{m\left(E_{n}(y)\right)}\|f\|_{\log } \beta^{n} \int_{E_{n}(y)} f d m \\
& \leq\left(1+C_{1}\right) c_{0}^{-1}\left(1+\beta^{n}\|f\|_{\log }\right) \int_{\Delta^{n}} f d m
\end{aligned}
$$

where in the last line we have used the fact that

$$
\sum_{y \in F^{-n} x} \int_{E_{n}(y)} f d m \leq \int_{\Delta^{n}} f d m .
$$

To modify Estimate \# 4, we need the following fact: if $\sum_{i} a_{i}$ and $\sum_{i} b_{i}$ are two series of positive terms, then

$$
\frac{\sum_{i} a_{i}}{\sum_{i} b_{i}} \leq \sup _{i} \frac{a_{i}}{b_{i}}
$$


Equation (3.3) becomes

$$
\begin{aligned}
\frac{\left|\mathcal{L}^{n} f(x)-\mathcal{L}^{n} f(y)\right|}{d(x, y) \mathcal{L}^{n} f(x)} \leq & \frac{\sum_{x^{\prime} \in F^{-n} x} g_{n}\left(y^{\prime}\right)\left|f\left(x^{\prime}\right)-f\left(y^{\prime}\right)\right| / d(x, y)}{\sum_{x^{\prime} \in F^{-n} x} g_{n}\left(x^{\prime}\right) f\left(x^{\prime}\right)} \\
& +\frac{\sum_{x^{\prime} \in F^{-n} x} f\left(x^{\prime}\right)\left|g_{n}\left(x^{\prime}\right)-g_{n}\left(y^{\prime}\right)\right| / d(x, y)}{\sum_{x^{\prime} \in F^{-n} x} g_{n}\left(x^{\prime}\right) f\left(x^{\prime}\right)} \\
\leq & \sup _{x^{\prime} \in F^{-n} x} \frac{g_{n}\left(y^{\prime}\right)}{g_{n}\left(x^{\prime}\right)} \frac{\left|f\left(x^{\prime}\right)-f\left(y^{\prime}\right)\right|}{d(x, y) f\left(x^{\prime}\right)}+\sup _{x^{\prime} \in F^{-n} x} \frac{\left|1-\left(g_{n}\left(y^{\prime}\right) / g_{n}\left(x^{\prime}\right)\right)\right|}{d(x, y)} \\
\leq & \left(1+C_{1}\right) \beta^{n}\|f\|_{\log }+C_{1} .
\end{aligned}
$$

Since $\|\cdot\|_{\log }$ is scale invariant, (3.5) implies for all $n \geq 0$

$$
\left\|\mathcal{L}_{1}^{n} f\right\|_{\log }=\left\|\mathcal{L}^{n} f\right\|_{\log } \leq\left(1+C_{1}\right) \beta^{n}\|f\|_{\log }+C_{1}
$$

Using (3.4),

$$
\frac{\left|\mathcal{L}^{n} f(x)\right|}{\left|\mathcal{L}^{n} f\right|_{1}} \leq \frac{\left(1+C_{1}\right) c_{0}^{-1}\left(1+\beta^{n}\|f\|_{\log }\right) \int_{\Delta^{n}} f d m}{\int_{\Delta^{n}} f d m} \leq\left(1+C_{1}\right) c_{0}^{-1}\left(1+\beta^{n}\|f\|_{\log }\right)
$$

so that the $\|\cdot\|_{\infty}$-norm stays bounded on the base of the tower. In order for this norm to remain bounded on successive levels, we need to ensure that $|\mathcal{L} f|_{1} \geq \beta$ for each $f \in \mathcal{B}_{M}$. Compute that

$$
\begin{aligned}
\int_{\Delta} \mathcal{L} f d m & =\int_{\Delta^{1}} f d m=1-\sum_{\ell \geq 1} \int_{\hat{F}^{-1} H_{\ell, j}} f d m \\
& \geq 1-\sum_{\ell \geq 1}\left\|f_{\ell-1, j}\right\|_{\infty} \beta^{-(\ell-1)} m\left(H_{\ell}\right) \\
& \geq 1-M \sum_{\ell \geq 1} \beta^{-(\ell-1)} m\left(H_{\ell}\right) .
\end{aligned}
$$

Recall that $q=\sum_{\ell \geq 1} \beta^{-(\ell-1)} m\left(H_{\ell}\right)$. Thus $|\mathcal{L} f|_{1}>\beta$ if $1-q M>\beta$ and $M$ must be chosen large enough so that $\mathcal{L}_{1}^{n}$ maps $\mathcal{B}_{M}$ back into itself for large enough $n$. Equations (3.4) and (3.5) require that we choose $M \in\left(\left(1+C_{1}\right) c_{0}^{-1},(1-\beta) q^{-1}\right)$. Thus $q<(1-\beta) / M<(1-\beta) c_{0} /\left(1+C_{1}\right)$ is a sufficient condition on the size of $H$ and is precisely assumption $(\mathrm{H} 1)$.

Proof of Proposition 2.4. The proof divides into several steps.

1. Quasi-compactness of $\mathcal{L}$. Proposition 3.1 implies that there exists $N \geq 1$ such that $\mathcal{L}_{1}^{N} \mathcal{B}_{M} \subset \mathcal{B}_{M}$. Since $\mathcal{L}_{1}^{N}$ is continuous on $\mathcal{B}_{M}$, which is a convex, compact subset of $L^{1}(m)$, the Schauder-Tychonoff theorem guarantees the existence of a fixed point $\varphi \in \mathcal{B}_{M}$, which is a conditionally invariant density for $\mathcal{L}^{N}$ with eigenvalue $\rho=\int_{\Delta^{N}} \varphi d m$.

Proposition 2.3 implies that the essential spectral radius of $\mathcal{L}^{N}$ is bounded by $\beta^{N}$ and Proposition 3.1 guarantees that $\rho>\beta^{N}$.

Thus $\mathcal{L}^{N}$ is quasi-compact with spectral radius at least $\rho$. We conclude that $\mathcal{L}$ is quasicompact with spectral radius at least $\lambda:=\rho^{1 / N}$ and essential spectral radius $\beta<\lambda$.

Let $N_{0} \leq N$ be the least positive integer such that $\mathcal{L}^{N_{0}} \varphi=\lambda^{N_{0}} \varphi$. In the next part of the proof, Steps $2-5$, we assume that $F$ is mixing and that $N_{0}=1$. These assumptions are removed in Steps 6 and 7. 
2. The density $\varphi$. We claim that there exists $\delta>0$ such that $\delta \leq \varphi \mid \Delta_{0} \leq \delta^{-1}$. It is then immediate from the conditional invariance condition $\lambda^{-1} \mathcal{L} \varphi=\varphi$ that $\delta \lambda^{-\ell} \leq \varphi \mid \Delta_{\ell} \leq$ $\delta^{-1} \lambda^{-\ell}$.

By conditional invariance, for $x \in \Delta_{\ell}, \varphi(x)=\lambda^{-\ell} \varphi\left(F^{-\ell} x\right)$, so that $\varphi \equiv 0$ on $\Delta$ if $\varphi \equiv 0$ on $\Delta_{0}$. Thus there exists $x \in \Delta_{0}$ such that $\varphi(x)>0$. Using conditional invariance once more, we obtain $x^{\prime} \in F^{-1} x$ such that $\varphi\left(x^{\prime}\right)>0$. Let $Z$ be the partition element containing $x^{\prime}$. Since $\varphi \in \mathcal{B}_{M}$, it follows that $\varphi \geq \kappa>0$ on $Z$. By construction, $F(Z) \supseteq Z^{\prime}$ for some $Z^{\prime} \in \mathcal{Z}_{0}^{i m}$. By conditional invariance, $\inf _{Z^{\prime}} \varphi \geq \lambda^{-1} \kappa \inf _{Z} g>0$. By transitivity, conditional invariance, and the property that $\varphi \in \mathcal{B}_{M}$, we obtain a similar lower bound for each $Z^{\prime} \in \mathcal{Z}_{0}^{i m}$. The claim follows from finiteness of the partition $\mathcal{Z}_{0}^{i m}$.

3. Spectral radius. Now suppose $f \in \mathcal{B}$ such that $\mathcal{L} f=\alpha f$ and $|\alpha|>\lambda$. Note that $f$ satisfies $f(x)=\alpha^{-\ell} f\left(F^{-\ell} x\right)$ for each $x \in \Delta_{\ell}, \ell \geq 1$. Since $\varphi \geq \delta$, there exists $K>0$ such that $K \varphi \geq|f|$ on $\Delta_{0}$. But since $f$ grows like $\alpha^{-\ell}$ and $\varphi$ grows like $\lambda^{-\ell}$ on level $\ell$, we have $K \varphi \geq|f|$ on $\Delta$. By the positivity of $\mathcal{L}, K \mathcal{L}^{n} \varphi \geq \mathcal{L}^{n}|f| \geq\left|\mathcal{L}^{n} f\right|$ for each $n$. But this implies that $K \lambda^{n} \varphi \geq|\alpha|^{n}|f|$ for each $n$. Since $\lambda<|\alpha|$, it follows that $f \equiv 0$. Hence $\mathcal{L}$ has spectral radius precisely $\lambda$.

4. Simplicity of $\lambda$. Suppose $f \in \mathcal{B}$ such that $\mathcal{L} f=\lambda f$. As in Step 3, we can choose $K>0$ such that $f+K \varphi>0$. Let $\psi=(f+K \varphi) / C>0$ where $C=|f|_{1}+K$ is chosen so that $\psi$ is a probability density. Define $\psi_{s}=s \varphi+(1-s) \psi$ and let $J=\{s \in \mathbb{R}$ : $\left.\inf _{\Delta} \psi_{s}>0\right\}$. Note that for $s \in J, \mathcal{L} \psi_{s}=\lambda \psi_{s}$ and $\left|\psi_{s}\right|_{1}=1$. Since $\psi_{s}$ is Lipschitz and bounded away from zero, $\left\|\psi_{s}\right\|_{\log }<\infty$. In fact, (3.6) implies that

$$
\left\|\psi_{s}\right\|_{\log }=\lim _{n \rightarrow \infty}\left\|\mathcal{L}_{1}^{n} \psi_{s}\right\|_{\log } \leq M,
$$

so that $\psi_{s} \in \mathcal{B}_{M}$ for all $s \in J$.

Since $\psi_{s}$ is conditionally invariant, the identity $\left.\psi_{s}\right|_{\Delta_{\ell}}=\left.\lambda^{-\ell} \psi_{s}\right|_{\Delta_{0}}$ implies that $\inf _{\Delta} \psi_{s}=\inf _{\Delta_{0}} \psi_{s}$, so that $J$ is open. Now let $t \in \partial J$. Since $\psi_{s} \in \mathcal{B}_{M}$ for all $s \in J$ and $\mathcal{B}_{M}$ is closed, we have $\psi_{t} \in \mathcal{B}_{M}$. If $\psi_{t}$ vanishes on $\Delta_{0}$, then $\psi_{t}$ vanishes on an entire element $Z^{\prime} \in \mathcal{Z}_{0}^{i m}$. Since $\psi_{t} \geq 0$, this implies that $\psi_{t} \equiv 0$ on all elements of $\mathcal{Z}_{0}^{i m}$ which map to $Z^{\prime}$ and by transitivity $\psi_{t}$ is zero on all of $\Delta$. Thus $\psi_{t}$ has strictly positive infimum on $\Delta_{0}$ and, since it is conditionally invariant, it must have the same infimum on $\Delta$. Thus $t \in J$, so $J$ is closed. Since $J$ is non-empty, $J=\mathbb{R}$, which is only possible if $f=c \varphi$ for some $c \in \mathbb{R}$.

It remains to eliminate generalized eigenvectors. Suppose $f \in \mathcal{B}$ such that $\mathcal{L} f=$ $\lambda(f+\varphi)$. Then $\mathcal{L}^{n} f=\lambda^{n} f+n \lambda^{n} \varphi=\lambda^{n} f+\mathcal{L}^{n}(n \varphi)$ so that $\mathcal{L}^{n}(f-n \varphi)=\lambda^{n} f$. This implies that for $x \in \Delta_{\ell}$,

$$
f(x)=\lambda^{-\ell}(f-\ell \varphi) \circ F^{-\ell}(x) .
$$

Since for $\ell$ large enough, $f-\ell \varphi<0$ on $\Delta_{0}$, we have $f<0$ on $\bigcup_{\ell \geq L} \Delta_{\ell}$ for some $L>0$.

Choose $K>0$ large such that $\psi:=f-K \varphi<0$ on $\bigcup_{\ell<L} \Delta_{\ell}$. Since $\psi<f$, we have $\psi<0$ on the whole of $\Delta$. For each $n \geq 0$,

$$
0>\lambda^{-n} \int_{\Delta^{n}} \psi d m=\lambda^{-n} \int_{\Delta} \mathcal{L}^{n} \psi d m=\int_{\Delta}(\psi+n \varphi) d m=\int_{\Delta} \psi d m+n,
$$

which is a contradiction.

5. Absence of peripheral spectrum. Suppose $f \in \mathcal{B},|f|_{1}=1$, such that $\mathcal{L} f=\alpha f$, where $\alpha=\lambda e^{i \omega}, \omega \in(0,2 \pi)$. We follow an approach similar to Step 4, modified to take 
into account the fact that $f$ is complex and $\alpha \neq \lambda$. Notice that by conditional invariance,

$$
\left.f\right|_{\Delta_{\ell}}=\left.\lambda^{-\ell} e^{-i \omega \ell} f\right|_{\Delta_{0}}
$$

so that $f$ grows like $\varphi$ plus a rotation up the levels of the tower.

Define $\psi=(\operatorname{Re}(f)+K \varphi) / C^{\prime}$, where $K$ is chosen large enough that $\psi>0$ and $C^{\prime}$ normalizes $|\psi|_{1}=1$. By replacing $f$ with $-f$ if necessary, we can guarantee that $\int_{\Delta} \operatorname{Re}(f) d m \leq 0$, so that $C^{\prime} \leq K$. Also notice that since $f$ and $\varphi$ grow at the same rate, there exists $\delta_{0}>0$ such that

$$
\delta_{0} \lambda^{-\ell} \leq \psi(x) \leq \delta_{0}^{-1} \lambda^{-\ell}
$$

for $x \in \Delta_{\ell}$.

As before, define $\psi_{s}=s \varphi+(1-s) \psi$ and let $J=\left\{s \in \mathbb{R}: \inf _{\Delta} \psi_{s}>0\right\}$. Owing to (3.8), $J$ is open. However, $\psi_{s}$ is not conditionally invariant since $\alpha \neq \lambda$ so the second part of the argument needs some modification.

Notice that

$$
\lambda^{-n} \mathcal{L}^{n} \psi=\left(\operatorname{Re}\left(e^{i \omega n} f\right)+K \varphi\right) / C^{\prime}
$$

so we may choose a sequence $n_{k}$ such that $\lambda^{-n_{k}} \mathcal{L}^{n_{k}} \psi \rightarrow \psi$ as $k \rightarrow \infty$. This implies also that $\lambda^{-n_{k}} \mathcal{L}^{n_{k}} \psi_{s} \rightarrow \psi_{s}$ along the same sequence and by (3.6) we have $\left\|\psi_{s}\right\|_{\text {log }} \leq M$ so that $\psi_{s} \in \mathcal{B}_{M}$ for $s \in J$. Now let $t>1$ be the right endpoint of $J$. Since $\mathcal{B}_{M}$ is closed, we have $\psi_{t} \in \mathcal{B}_{M}$. The rest of Step 5 relies on the following lemma.

LEMMA 3.2. $\psi_{t}$ is bounded away from 0 .

It is easy to see that the lemma completes the proof of Step 5 since then $t \in J$ and we conclude that $J \supset \mathbb{R}^{+}$. Now $\psi_{s}>0$ for all $s>0$, implies $\varphi>\psi$. Thus

$$
\varphi>(\operatorname{Re}(f)+K \varphi) / C^{\prime} \Rightarrow\left(C^{\prime}-K\right) \varphi>\operatorname{Re}(f) \Rightarrow 0>\operatorname{Re}(f)
$$

since $C^{\prime} \leq K$. But $\operatorname{Re}(f)$ must change sign on $\Delta$ owing to the rotation as we move up the levels of the tower given by (3.7). This contradicts the existence of $\alpha$.

Proof of Lemma 3.2. Since $\left|\psi_{t}\right|_{1}=1$ and $\psi_{t} \geq 0$, there exists $\ell \geq 0$ and $x \in \Delta_{\ell}$ such that $\psi_{t}(x)>0$. Since $\lambda^{-n_{k}} \mathcal{L}^{n_{k}} \psi_{t} \rightarrow \psi_{t}$, there exists $k$ with $n_{k}>\ell$ such that $\lambda^{-n_{k}} \mathcal{L}^{n_{k}} \psi_{t}(x)$ $>0$. Hence there is a preimage $x^{\prime} \in F^{-n_{k}}(x)$ such that $\psi_{t}\left(x^{\prime}\right)>0$. Let $Z_{1} \in \mathcal{Z}$ be the partition element containing $x^{\prime}$. By construction, $Z_{1}$ does not iterate into a hole before reaching $\Delta_{0}$ (in $m=n_{k}-\ell$ iterates). In particular, $F^{m} Z_{1}$ covers an element of $\mathcal{Z}_{0}^{i m}$. Since $F$ is mixing, there exists an $N_{1}>0$ such that for each $n \geq N_{1}, F^{n} Z_{1} \supset \Delta_{0}$.

Since $\psi_{t} \in \mathcal{B}_{M}$, it follows that $\inf _{Z_{1}} \psi_{t}=: \kappa>0$. Note that for any $n \geq 0$, the definition of $\psi_{t}$ and equation (3.9) imply that

$$
\begin{aligned}
\lambda^{-n} \mathcal{L}^{n} \psi_{t} & =t \varphi+(1-t)\left[\operatorname{Re}\left(e^{i \omega n} f\right)+K \varphi\right] / C^{\prime} \\
& =\psi_{t}+(1-t) \operatorname{Re}\left(\left(e^{i \omega n}-1\right) f\right) / C^{\prime} .
\end{aligned}
$$

Choose $\varepsilon<\kappa C^{\prime} /\left(2|1-t|\|f\|_{\infty}\right)$ and define $Q_{\varepsilon}=\left\{n \in \mathbb{N}:\left|e^{i \omega n}-1\right|<\varepsilon\right\}$. Notice that $Q_{\varepsilon}$ has bounded gaps, i.e., there exists a $K_{1}=K_{1}(\varepsilon)$ such that for any $n \geq K_{1}$, there is a $k \leq K_{1}$ such that $n-k \in Q_{\varepsilon}$. 
It is clear from (3.10) that

$$
\lambda^{-n} \mathcal{L}^{n} \psi_{t}(x) \geq \psi_{t}(x)-\varepsilon|1-t|\|f\|_{\infty} / C^{\prime} \geq \kappa / 2
$$

for $n \in Q_{\varepsilon}$ and $x \in Z_{1}$.

Fix $n \geq N_{1}+K_{1}$ and choose $k$ such that $N_{1} \leq k \leq N_{1}+K_{1}$ and $n-k \in Q_{\varepsilon}$. Note that for any $\ell, \inf _{\Delta_{\ell}} g$ may be zero if there are infinitely many $Z \subset \Delta_{\ell}$ with $R(Z)=1$. However, since we only require returns to finitely many $Z^{\prime} \in \mathcal{Z}_{0}^{i m}$ for finitely many times, $N_{1} \leq k \leq N_{1}+K_{1}$, we may choose a set $W \subset \bigcup_{\ell \leq N_{1}+K_{1}} \Delta_{\ell}$ containing only finitely many $Z$ such that for each $x \in \Delta_{0}$ there is a point $y_{1} \in Z_{1}$ such that $F^{k} y_{1}=x$ and $F^{i} y_{1} \in W$ for $0 \leq i \leq k-1$.

Now using (3.11) and the fact that $n-k \in Q_{\varepsilon}$, we estimate

$$
\begin{aligned}
\lambda^{-n} \mathcal{L}^{n} \psi_{t}(x) & =\lambda^{-k} \mathcal{L}^{k}\left(\lambda^{k-n} \mathcal{L}^{n-k} \psi_{t}\right)(x)=\lambda^{-k} \sum_{F^{k} y=x} \lambda^{k-n} \mathcal{L}^{n-k} \psi_{t}(y) g_{k}(y) \\
& \geq \lambda^{-k}\left(\lambda^{k-n} \mathcal{L}^{n-k} \psi_{t}\right)\left(y_{1}\right) g_{k}\left(y_{1}\right) \geq \frac{1}{2} \lambda^{-N_{1}} \inf _{W} g_{N_{1}+K_{1}}=: \kappa^{\prime}>0 .
\end{aligned}
$$

Thus $\inf _{\Delta_{0}} \lambda^{-n} \mathcal{L}^{n} \psi_{t} \geq \kappa^{\prime}$ for all $n \geq N_{1}+K_{1}$.

Now on $\Delta_{\ell}$, for $n \geq \ell+N_{1}+K_{1}$,

$$
\lambda^{-n} \mathcal{L}^{n} \psi_{t}(x)=\lambda^{-\ell} \lambda^{\ell-n} \mathcal{L}^{n-\ell} \psi_{t}\left(F^{-\ell} x\right) \geq \lambda^{-\ell} \kappa^{\prime} .
$$

Therefore for large $n, \inf _{\Delta_{\ell}} \lambda^{-n} \mathcal{L}^{n} \psi_{t} \geq \kappa^{\prime}$ for all $\ell \leq n-N_{1}-K_{1}$. Since $\psi_{t}=$ $\lim _{k} \lambda^{-n_{k}} \mathcal{L}^{n_{k}} \psi_{t}$, we have $\inf _{\Delta} \psi_{t} \geq \kappa^{\prime}$.

6. Mixing implies $N_{0}=1$. Suppose that $\mathcal{L}^{N_{0}} \varphi=\lambda^{N_{0}} \varphi$. The proofs of Steps 2 and 4 go through with $\mathcal{L}$ replaced by $\mathcal{L}^{N_{0}}$, implying that $\lambda^{N_{0}}$ is a simple eigenvalue for $\mathcal{L}^{N_{0}}$. (The proofs are modified in the obvious way. For example, $\Delta_{0}$ is replaced by $\Delta_{0} \cup \cdots \cup \Delta_{N_{0}-1}$ and mixing is used instead of transitivity.) However $\mathcal{L}^{N_{0}}(\mathcal{L} \varphi)=\lambda^{N_{0}} \mathcal{L} \varphi$, so we deduce that $\mathcal{L} \varphi=c \varphi$ for some $c \in \mathbb{R}$, with $c^{N_{0}}=\lambda^{N_{0}}$. Positivity of $\mathcal{L}$ implies that $c>0$, so $c=\lambda$. Hence $\mathcal{L} \varphi=\lambda \varphi$, that is $N_{0}=1$.

7. Non-mixing case. First suppose that $F$ is transitive with period $p$. Then $F^{p}$ has $p$ distinct components in $\Delta$ and is mixing on each of them. Applying (i) to $\mathcal{L}^{p}$ implies that $\lambda^{p}$ is an eigenvalue of algebraic and geometric multiplicity $p$ and there are no further eigenvalues on or outside the circle of radius $\lambda^{p}$. The corresponding eigenvalues for $\mathcal{L}$ lie at $p$ th roots of $\lambda^{p}$, and it follows easily from transitivity that all $p$ th roots are realized by simple eigenvalues, proving (ii).

Finally, since $\mathcal{L}$ is quasi-compact, there are only finitely many transitive components of $\Delta$. Restricting to a single component, (iii) reduces to the transitive case (ii).

3.3. An invariant measure on $\Delta^{\infty}$. Proof of Proposition 2.8. We assume that $F$ is mixing and as usual denote by $\varphi$ the unique eigenvector with eigenvalue $\lambda$. We divide the proof into three parts.

(i) Existence of $\nu$. Let $f \in \mathcal{B}_{0}$. Since $\left.\varphi\right|_{\Delta_{\ell}} \sim \lambda^{-\ell}$ where $\lambda>\beta$, it follows from the definitions of $\mathcal{B}$ and $\mathcal{B}_{0}$ that $\varphi f \in \mathcal{B}$. By Corollary 2.5,

$$
\mathcal{F}(f):=\lim _{n \rightarrow \infty} \lambda^{-n} \varphi^{-1} \mathcal{L}^{n}(\varphi f)=c(\varphi f) .
$$


Hence (3.12) defines a linear functional $\mathcal{F}: \mathcal{B}_{0} \rightarrow \mathbb{R}$. We also have

$$
\left|\mathcal{L}^{n}(\varphi f)\right| \leq|f|_{\infty} \mathcal{L}^{n} \varphi=|f|_{\infty} \lambda^{n} \varphi
$$

so that $|\mathcal{F}(f)| \leq|f|_{\infty}$.

Since $\mathcal{F}$ is a bounded linear functional on $\mathcal{B}_{0}$, there exists a measure $v$ such that $\mathcal{F}(f)=\int f d v$ for each $f$ in $\mathcal{B}_{0}$. Since $\mathcal{F}(1)=1, v$ is a probability measure. Notice also that we can write $\lambda^{-n} \mathcal{L}^{n}(\varphi f) \rightarrow \varphi v(f)$ where convergence takes place in $\mathcal{B}$ and hence in $L^{1}(m)$. Since $\int_{\Delta} \varphi d m=1$, it follows that

$$
\nu(f)=\lim _{n \rightarrow \infty} \lambda^{-n} \int_{\Delta} \mathcal{L}^{n}(\varphi f) d m=\lim _{n \rightarrow \infty} \lambda^{-n} \int_{\Delta^{n}} f \varphi d m=\lim _{n \rightarrow \infty} \lambda^{-n} \int_{\Delta^{n}} f d \mu
$$

so that $v$ is supported on $\Delta^{\infty}$. Also, from (3.12) it follows that $c(f)=v\left(\varphi^{-1} f\right)$ for each $f \in \mathcal{B}_{0}$.

Note that $\mathcal{L}(\varphi f \circ F)=f \mathcal{L} \varphi=\lambda \varphi f$ and so

$$
\begin{aligned}
\mathcal{F}(f \circ F) & =\lim _{n \rightarrow \infty} \lambda^{-n} \varphi^{-1} \mathcal{L}^{n}(\varphi f \circ F)=\lim _{n \rightarrow \infty} \lambda^{-n} \varphi^{-1} \mathcal{L}^{n-1}(\lambda \varphi f) \\
& =\lim _{n \rightarrow \infty} \lambda^{-(n-1)} \varphi^{-1} \mathcal{L}^{n-1}(\varphi f)=\mathcal{F}(f) .
\end{aligned}
$$

Hence $v$ is an invariant measure for $F$ (and $\hat{F}$, since $F=\hat{F}$ on $\Delta^{\infty}$ ).

(ii) $v$ is ergodic. Since $F$ is transitive on $\mathcal{Z}_{0}^{i m}$, given $Z_{1}^{\prime}, Z_{2}^{\prime} \in \mathcal{Z}_{0}^{i m}$, we may choose $n \in \mathbb{N}$ such that $F^{n}\left(Z_{1}^{\prime}\right) \supseteq Z_{2}^{\prime}$. Since $\Delta^{\infty}$ is an $F$-invariant set, this implies that $F^{n}\left(Z_{1}^{\prime} \cap \Delta^{\infty}\right) \supseteq Z_{2}^{\prime} \cap \Delta^{\infty}$. So $\left.F\right|_{\Delta^{\infty}}$ is transitive.

Let $Z_{i}^{n} \subset \Delta^{\infty}$ denote a cylinder set of length $n$ with respect to the partition $\mathcal{Z}_{0} \cap \Delta^{\infty}$. Now suppose $A=\bigcup_{i, n} Z_{i}^{n}$ is a countable union of such cylinder sets with $F^{-1} A=A$ and $v(A)>0$. Since $A$ is a countable union, we must have $v\left(Z_{i}^{n}\right)>0$ for some $i$ and $n$. This implies that $F^{n}\left(Z_{i}^{n}\right)=Z \cap \Delta^{\infty}$ for some $Z \in \mathcal{Z}_{0}$, and $F^{n+R(Z)}\left(Z_{i}^{n}\right) \supseteq Z^{\prime} \cap \Delta^{\infty}$ for some $Z^{\prime} \in \mathcal{Z}_{0}^{i m}$. In particular, $Z^{\prime} \cap \Delta^{\infty} \subset A$. Since $F$ is transitive on $\Delta^{\infty}, \bigcup_{k \geq 0} F^{k}\left(Z^{\prime} \cap\right.$ $\left.\Delta^{\infty}\right)=\Delta^{\infty}$. Thus $A=\Delta^{\infty}$ so $v(A)=1$.

Since $\mathcal{Z}$ is a generating partition on $\Delta^{\infty}$, we conclude that $v$ is ergodic.

(iii) Exponential decay of correlations. Let $f_{1}, f_{2} \in \mathcal{B}_{0}$. Recall that $\nu\left(f_{1}\right)=c\left(f_{1} \varphi\right)$. By definition of $v$,

$$
\begin{aligned}
& \int_{\Delta^{\infty}} f_{1} f_{2} \circ F^{n} d v-v\left(f_{1}\right) v\left(f_{2}\right) \\
& =\lim _{k \rightarrow \infty} \lambda^{-k} \int_{\Delta^{k}} f_{1} f_{2} \circ F^{n} \varphi d m-\int_{\Delta^{\infty}} v\left(f_{1}\right) f_{2} d v \\
& =\lim _{k \rightarrow \infty} \lambda^{-k} \int_{\Delta^{k-n}} \mathcal{L}^{n}\left(f_{1} \varphi\right) f_{2} d m-\lim _{k \rightarrow \infty} \lambda^{n-k} \int_{\Delta^{k-n}} v\left(f_{1}\right) f_{2} \varphi d m \\
& =\lim _{k \rightarrow \infty} \lambda^{n-k} \int_{\Delta^{k-n}}\left[\lambda^{-n} \mathcal{L}^{n}\left(f_{1} \varphi\right)-c\left(f_{1} \varphi\right) \varphi\right] f_{2} d m \\
& =\lim _{k \rightarrow \infty} \lambda^{-k} \sum_{\ell \geq 0} \int_{\Delta^{k} \cap \Delta_{\ell}}\left[\lambda^{-n} \mathcal{L}^{n}\left(f_{1} \varphi\right)-c\left(f_{1} \varphi\right) \varphi\right] f_{2} d m
\end{aligned}
$$


Recall that $f_{1} \varphi \in \mathcal{B}$. For $F$ mixing, it follows from Corollary 2.5 that

$$
\begin{aligned}
& \left|\int_{\Delta^{k} \cap \Delta_{\ell}}\left[\lambda^{-n} \mathcal{L}^{n}\left(f_{1} \varphi\right)-c\left(f_{1} \varphi\right) \varphi\right] f_{2} d m\right| \\
& \quad \leq\left|1_{\Delta_{\ell}}\left(\lambda^{-n} \mathcal{L}^{n}\left(f_{1} \varphi\right)-c\left(f_{1} \varphi\right) \varphi\right)\right|_{\infty}\left|f_{2}\right|_{\infty} m\left(\Delta^{k} \cap \Delta_{\ell}\right) \\
& \quad \leq\left\|\lambda^{-n} \mathcal{L}^{n}\left(f_{1} \varphi\right)-c\left(f_{1} \varphi\right) \varphi\right\| \beta^{-\ell}\left|f_{2}\right|_{\infty} m\left(\Delta^{k} \cap \Delta_{\ell}\right) \\
& \quad \leq C\left\|f_{1} \varphi\right\|\left|f_{2}\right|_{\infty} \sigma^{n} \beta^{-\ell} m\left(\Delta^{k} \cap \Delta_{\ell}\right) .
\end{aligned}
$$

Hence

$$
\begin{aligned}
\left|\int_{\Delta^{\infty}} f_{1} f_{2} \circ F^{n} d v-v\left(f_{1}\right) v\left(f_{2}\right)\right| & \leq \lim _{k \rightarrow \infty} \lambda^{-k} \sum_{\ell \geq 0} C\left\|f_{1} \varphi\right\|\left|f_{2}\right|_{\infty} \sigma^{n} \beta^{-\ell} m\left(\Delta^{k} \cap \Delta_{\ell}\right) \\
& =C\left\|f_{1} \varphi\right\|\left|f_{2}\right|_{\infty} \sigma^{n} \lim _{k \rightarrow \infty} \lambda^{-k} \int_{\Delta^{k}} f_{\beta} d m,
\end{aligned}
$$

where $\left.f_{\beta}\right|_{\Delta_{\ell}}:=\beta^{-\ell}$. In particular, $f_{\beta} \in \mathcal{B}$. By Corollary $2.5, \lambda^{-k} \mathcal{L}^{k} f_{\beta}$ converges to $c\left(f_{\beta}\right) \varphi$ in $\mathcal{B}$ and hence in $L^{1}(m)$ so that $\lim _{k \rightarrow \infty} \lambda^{-k} \int_{\Delta^{k}} f_{\beta} d m=c\left(f_{\beta}\right)$, completing the proof.

3.4. Escape rates from $\Delta$. Notice that the functional analytic approach adopted thus far only tells us that $\lambda$ represents the slowest rate of escape from $\Delta$ for elements of $\mathcal{B}$, but in general there are functions which escape at faster rates. The estimates on the functional $\mathcal{F}$ in $\$ 3.3$ and the existence of the invariant measure $v$ allow us to establish the uniformity of escape rates for certain functions in $\mathcal{B}$. Since the indicator functions of elements of the partition $\mathcal{Z}$ are in this space, we also obtain uniform escape rates of mass from certain sets and in particular for the reference measure $m$ on the tower.

Proposition 3.3. Let $F$ be mixing and satisfy properties (P1)-(P3) and (H1) of \$2.1.1. For each $f \in \mathcal{B}_{0}$ with $f \geq 0$, we have $v(f)>0$ if and only if

$$
\lim _{n \rightarrow \infty} \frac{\mathcal{L}^{n} f}{\left|\mathcal{L}^{n} f\right|_{1}}=\varphi
$$

where as usual, the convergence is in the $\|\cdot\|$-norm. In particular, the reference measure converges to the a.c.c.i.m.

Proof. By Proposition 2.6, equation (3.13) holds if and only if $c(f)>0$. Thus it suffices to prove $v(f)>0$ if and only if $c(f)>0$.

Note that from the proof of Proposition 2.8, $v(f)=c(\varphi f) \geq \delta c(f)$ since $\varphi \geq \delta$. So $c(f)>0$ implies $v(f)>0$ immediately.

Now fix $f \in \mathcal{B}_{0}$ and suppose $v(f)>0$. Let $\Delta_{\ell}^{n}=\Delta_{\ell} \cap \Delta^{n}$ be the subset of $\Delta_{\ell}$ which has not escaped by time $n$. Set $\Delta_{(K)}^{n}=\bigcup_{\ell=0}^{K} \Delta_{\ell}^{n}$ and $\Delta_{+}^{n}=\Delta^{n} \backslash \Delta_{(K)}^{n}$.

For $\varepsilon \in(0,1)$, choose $K$ such that $v\left(\Delta_{+}^{0}\right)|f|_{\infty}<\varepsilon v(f)$. Then

$$
\begin{aligned}
v(f) & =\lim _{n \rightarrow \infty}\left(\lambda^{-n} \int_{\Delta_{(K)}^{n}} f d \mu+\lambda^{-n} \int_{\Delta_{+}^{n}} f d \mu\right) \\
& \leq \lim _{n \rightarrow \infty}\left(\lambda^{-n} \lambda^{-K} \delta^{-1} \int_{\Delta_{(K)}^{n}} f d m+\lambda^{-n}|f|_{\infty} \int_{\Delta_{+}^{n}} d \mu\right) \\
& \leq \lambda^{-K} \delta^{-1} c(f)+|f|_{\infty} v\left(\Delta_{+}^{0}\right) \leq \lambda^{-K} \delta^{-1} c(f)+\varepsilon v(f) .
\end{aligned}
$$

Since $\varepsilon \in(0,1)$, we have $c(f)>0$. 
Since $v(1)=1$, the normalized push forward of the reference measure $m$ converges to $\mu$ as $n \rightarrow \infty$.

COROLlary 3.4. Let $A=\bigcup_{(\ell, j) \in J} Z_{\ell, j}$ be a union of partition elements such that $v(A)>0$. Then there exists $C>0$ such that

$$
C^{-1} \lambda^{n} \leq m\left(\Delta^{n} \cap A\right) \leq C \lambda^{n}
$$

for each $n \in \mathbb{N}$ so that mass with respect to $m$ escapes from A at a uniform rate matching that of the conditionally invariant measure.

Proof. First note that for any $f \in \mathcal{B}$, we have

$$
\left|\mathcal{L}^{n} f\right|_{1} \leq\left\|\mathcal{L}^{n} f\right\| \sum_{\ell \geq 0} \beta^{-\ell} m\left(\Delta_{\ell}\right) \leq C\left\|\mathcal{L}^{n}\right\|\|f\| \leq C \lambda^{n}\|f\|,
$$

so that the upper bound in (3.14) is trivial.

Let $\chi_{A}$ be the indicator function for $A$ and notice that $\chi_{A} \in \mathcal{B}_{0}$. Integrating the limit in Corollary 2.5 , we get

$$
c\left(\chi_{A}\right)=\lim _{n \rightarrow \infty} \lambda^{-n} \int_{\Delta} \mathcal{L}^{n} \chi_{A} d m=\lim _{n \rightarrow \infty} \lambda^{-n} \int_{\Delta^{n}} \chi_{A} d m .
$$

Since $c\left(\chi_{A}\right)>0$ by Proposition 3.3 and $m\left(\Delta^{n} \cap A\right)$ forms a decreasing sequence, there must exist a $C>0 \operatorname{such} \lambda^{-n} m\left(\Delta^{n} \cap A\right) \geq C^{-1}$ for all $n$.

COROLlary 3.5. Let $Z=Z_{\ell, j}$ be a cylinder set and let $n>R(Z)$. There exists a constant $C>0$, independent of $Z$, such that if $\Delta^{n} \cap Z \neq \emptyset$, then

$$
C^{-1} \lambda^{n-R} m(Z) \leq m\left(\Delta^{n} \cap Z\right) \leq C \lambda^{n-R} m(Z) .
$$

Proof. By bounded distortion, we have

$$
m\left(\Delta^{n} \cap Z\right)\left|\left(F^{R}\right)^{\prime}(y)\right|=m\left(\Delta^{n-R} \cap F^{R} Z\right) \text { for some } y \in Z .
$$

Since $F^{R} Z=Z^{\prime}$ for some $Z^{\prime} \in \mathcal{Z}_{0}^{i m}$ and $\mathcal{Z}_{0}^{i m}$ is finite, by Corollary 3.4, we can find $C$ independent of $Z^{\prime}$ such that

$$
C^{-1} \lambda^{n} \leq m\left(\Delta^{n-R} \cap Z^{\prime}\right) \leq C \lambda^{n} .
$$

We complete the proof by noting that $\left|\left(F^{R}\right)^{\prime}(y)\right| \approx c_{0} / m(Z)$.

Corollary 3.6. Let $f \in \mathcal{B}, f \geq 0$, such that $v(x \in \Delta: f(x)>0)>0$. Then

$$
\lim _{n \rightarrow \infty} \frac{\mathcal{L}^{n} f}{\left|\mathcal{L}^{n} f\right|_{1}}=\varphi \text {. }
$$

Proof. Let $h=\min \{f, 1\}$ and note that $h \in \mathcal{B}_{0}$. Also $v(h)>0$ by assumption on $f$ since $h$ and $f$ share the same support. Thus $c(h)>0$ by Proposition 3.3. Now

$$
c(f)=\lim _{n \rightarrow \infty} \lambda^{-n} \int_{\Delta^{n}} f d m \geq \lim _{n \rightarrow \infty} \lambda^{-n} \int_{\Delta^{n}} h d m=c(h)>0
$$

so the limit for $f$ holds by Proposition 2.6. 


\section{Applications}

4.1. General approach. We set up our notation as follows. Let $\hat{T}$ be a piecewise $C^{1+\alpha}$ self-map of a metric space $(\hat{X}, \tilde{d})$ with open hole $\tilde{H}$. Let $\tilde{m}$ be a probability measure on $\hat{X}$ and let $\tilde{g}=d \tilde{m} / d(\tilde{m} \circ \hat{T})$. Suppose that a tower $(\hat{F}, \hat{\Delta})$ with hole $H$ and the properties of $\S 2.1 .1$ can be constructed over a reference set $\Lambda$. This implies that there exists a countable partition $\mathcal{Z}_{0}$ of $\Lambda$, a coarser partition $\mathcal{Z}_{0}^{i m}$, also of $\Lambda$, and a return time function $R$ which is constant on elements of $\mathcal{Z}_{0}$ and for which $\hat{T}^{R}(Z) \in \mathcal{Z}_{0}^{i m}$ or $\hat{T}^{R}(Z) \subset \tilde{H}$ for each $Z \in \mathcal{Z}_{0}$. The set $\Lambda$ is identified with $\hat{\Delta}_{0}$, and each level $\hat{\Delta}_{\ell}$ is associated with $\bigcup_{R(Z)>\ell} \hat{T}^{\ell}(Z)$. This defines a natural projection $\pi: \hat{\Delta} \rightarrow \hat{X}$ so that $\pi \circ \hat{F}^{n}=\hat{T}^{n} \circ \pi$ for each $n$. In general, we may choose $\Lambda$ so that $\Lambda \cap H=\emptyset$.

Following our previous notation, we define $X=\hat{X} \backslash \tilde{H}$ and $X^{n}=\bigcap_{i=0}^{n} \hat{T}^{-i} X$. The restricted maps are then $F^{n}=\left.\hat{F}^{n}\right|_{\Delta_{n}}$ on the tower and $T^{n}=\left.\hat{T}^{n}\right|_{X^{n}}$ on the underlying space.

We use the reference measure $\tilde{m}$ on $\hat{X}$ to define a reference measure $m$ on $\hat{\Delta}$ by letting $\left.m\right|_{\hat{\Delta}_{0}}=\left.\tilde{m}\right|_{\Lambda}$ and then simply defining $m$ on subsequent levels by $m(A)=m\left(\hat{F}^{-\ell} A\right)$ for measurable $A \subset \hat{\Delta}_{\ell}$. As before, we let $g=d m / d(m \circ \hat{F})$.

Given a measure $\mu$ on $\hat{\Delta}$, we define its projection $\tilde{\mu}$ onto $\hat{X}$, by $\tilde{\mu}=\pi_{*} \mu$. In terms of densities, this implies that if $d \mu=f d m$, then for almost every $u \in \hat{X}$ the density $\tilde{f}$ of $\tilde{\mu}$ is given by

$$
\mathcal{P}_{\pi} f(u)=\sum_{x \in \pi^{-1} u} f(x) / J \pi(x)
$$

where $J \pi=d(\tilde{m} \circ \pi) / d m$. Note that $\left|\mathcal{P}_{\pi} f\right|_{L^{1}(X, \tilde{m})}=|f|_{L^{1}(\Delta, m)}$. Since Radon-Nikodym derivatives multiply, we have

$$
\tilde{g}_{n}(\pi y) / J \pi(y)=g_{n}(y) / J \pi\left(\hat{F}^{n} y\right)
$$

for almost every $y \in \hat{\Delta}$ and each $n \geq 0$. This in turn implies that

$$
\mathcal{P}_{\pi}\left(\mathcal{L}_{F}^{n} f\right)=\mathcal{L}_{T}^{n}\left(\mathcal{P}_{\pi} f\right)
$$

for $f \in L^{1}(\Delta)$.

The importance of these relations lies in the fact that if $\varphi$ satisfies $\mathcal{L}_{F} \varphi=\lambda \varphi$ and $\tilde{f}=\mathcal{P}_{\pi} f$, then

$$
\frac{\mathcal{L}_{F}^{n} f}{\left|\mathcal{L}_{F}^{n} f\right|_{1}} \rightarrow \varphi \quad \text { in } L^{1}(m) \quad \text { implies } \quad \frac{\mathcal{L}_{T}^{n} \tilde{f}}{\left|\mathcal{L}_{T}^{n} \tilde{f}\right|_{1}} \rightarrow \mathcal{P}_{\pi} \varphi=: \tilde{\varphi} \quad \text { in } L^{1}(\tilde{m})
$$

and $\tilde{\varphi}$ satisfies $\mathcal{L}_{T} \tilde{\varphi}=\mathcal{P}_{\pi}\left(\mathcal{L}_{F} \varphi\right)=\lambda \tilde{\varphi}$ so that $\tilde{\varphi}$ defines a conditionally invariant measure for $T$ with the same eigenvalue as $\varphi$.

However, the space $\mathcal{P}_{\pi} \mathcal{B}$ is not well understood and functions in $\mathcal{P}_{\pi} \mathcal{B}$ are a priori no better than $L^{1}$. It is not even clear that the constant function corresponding to the original reference measure $\tilde{m}$ is in $\mathcal{P}_{\pi} \mathcal{B}$. Getting a handle on a nice class of functions in $\mathcal{P}_{\pi} \mathcal{B}$ is necessary for showing in particular applications that, for example, Lebesgue measure converges to the a.c.c.i.m. according to the results of the previous section.

In what follows, we identify two properties, (A1) and (A2), that guarantee $C^{\bar{\alpha}}(X) \subset$ $\mathcal{P}_{\pi} \mathcal{B}$ where $\bar{\alpha}$ depends on the smoothness and average expansion of $T$. (A1) is standard 
in constructions of Young towers and (A2) can be achieved with no added restrictions on the map or types of holes allowed. In $\S \S 4.2$ and 4.3 , we prove that the towers we construct have these properties.

Let $R_{n}(x)=R_{n-1}\left(T^{R(x)}(x)\right)$ be the $n$th good return of $x$ to $\Lambda$, for $n \geq 1$.

(A1) There exist constants $\tau>1$ and $C_{2}, C_{3}>0$ such that the following hold.

(a) For any $x \in \Lambda, n \geq 1$ and $k<R_{n}(x),\left|D T^{R_{n}(x)-k}\left(T^{k} x\right)\right|>C_{2} \tau^{R_{n}(x)-k}$.

(b) Let $x, y \in Z_{0, j}$ and $R=R\left(Z_{0, j}\right)$. Then $\left|\tilde{g}_{\ell}(\pi x) / \tilde{g}_{\ell}(\pi y)\right| \leq C_{3}$ for $\ell \leq R$. If $T^{R}\left(Z_{0, j}\right) \subseteq \Lambda$, then

$$
\left|\frac{\tilde{g}_{R}(\pi x)}{\tilde{g}_{R}(\pi y)}-1\right| \leq C_{3} d\left(T^{R}(\pi x), T^{R}(\pi y)\right)^{\alpha} .
$$

Property (A1)(a) says that although $T$ may not be expanding everywhere in its phase space, we only count returns to $\Lambda$ during which average expansion has occurred. Property (A1)(b) is simply bounded distortion. In fact, (A1) implies the distortion bound (2.1) as well as (P2) in the towers we use.

4.1.1. Lifting Hölder functions on $X$. Recall that $\tilde{d}$ is the metric on $X$ and $d$ is the symbolic metric on $\Delta$ defined in $\$ 2.1 .1$. Under assumption (A1)(a), these two metrics are compatible in the following sense.

LEMMA 4.1. For any $\bar{\alpha} \geq-\log \beta / \log \tau$, let $\tilde{f} \in C^{\bar{\alpha}}(X)$ and define $f$ on $\Delta$ by $f(x)=$ $\tilde{f}(\pi x)$ for each $x \in \Delta$. Then $f \in \mathcal{B}_{0}$ and $\|f\|_{0} \leq C_{2}^{-1}|\tilde{f}|_{C^{\bar{\alpha}} \text {. }}$

Proof. First we show that $\operatorname{Lip}(f)=\sup _{\ell, j} \operatorname{Lip}\left(f_{\ell, j}\right)<\infty$. Let $x, y \in Z_{\ell, j}$ and let $\tilde{x}=\pi x$ and $\tilde{y}=\pi y$. Then

$$
\frac{|f(x)-f(y)|}{d(x, y)}=\frac{|\tilde{f}(\tilde{x})-\tilde{f}(\tilde{y})|}{\tilde{d}(\tilde{x}, \tilde{y})^{\bar{\alpha}}} \cdot \frac{\tilde{d}(\tilde{x}, \tilde{y})^{\bar{\alpha}}}{d(x, y)} \leq C_{\bar{\alpha}, \tilde{f}} \frac{\tilde{d}(\tilde{x}, \tilde{y})^{\bar{\alpha}}}{d(x, y)} .
$$

Note that $d(x, y)=\beta^{s(x, y)}$ and that $s(x, y)$ is a return time for $\tilde{x}$ and $\tilde{y}$ so that $\left|D T^{s(x, y)}\right| \geq$ $C_{2} \tau^{s(x, y)}$ on $\pi\left(Z_{\ell, j}\right)$ by Property (A1)(a). Thus

$$
\tilde{d}(\tilde{x}, \tilde{y})=\frac{\tilde{d}(\tilde{x}, \tilde{y})}{\tilde{d}\left(T^{s(x, y)}(\tilde{x}), T^{s(x, y)}(\tilde{y})\right)} \tilde{d}\left(T^{s(x, y)}(\tilde{x}), T^{s(x, y)}(\tilde{y})\right) \leq C_{2}^{-1} \tau^{-s(x, y)} \operatorname{diam}(\Lambda) .
$$

This, together with (4.4), implies that $\operatorname{Lip}(f)<\infty$ since $\beta \geq \tau^{-\bar{\alpha}}$. Also $|f|_{\infty}=|\tilde{f}|_{\infty}$ $<\infty$, so $f \in \mathcal{B}_{0}$.

The problem is that in general $\mathcal{P}_{\pi}(\tilde{f} \circ \pi) \neq \tilde{f}$, so Lemma 4.1 does not imply that $C^{\bar{\alpha}}(X) \subset \mathcal{P}_{\pi} \mathcal{B}$ immediately.

4.1.2. A lift compatible with $\mathcal{P}_{\pi}$. Given $\tilde{f} \in C^{\bar{\alpha}}(X)$, we want to construct $f \in \mathcal{B}$ so that $\mathcal{P}_{\pi} f=\tilde{f}$. To do this, it is sufficient to have the following property on the tower constructed above the reference set $\Lambda$.

(A2) There exists an index set $J \subset \mathbb{N} \times \mathbb{N}$ such that the following hold.

(a) $\tilde{m}\left(X \backslash \bigcup_{(\ell, j) \in J} \pi\left(Z_{\ell, j}\right)\right)=0$.

(b) $\pi\left(Z_{\ell_{1}, j_{1}}\right) \cap \pi\left(Z_{\ell_{2}, j_{2}}\right)=\emptyset$ for all but finitely many $\left(\ell_{1}, j_{1}\right),\left(\ell_{2}, j_{2}\right) \in J$.

(c) Define $J \pi_{\ell, j}:=\left.J \pi\right|_{Z_{\ell, j}}$. Then $\sup _{(\ell, j) \in J}\left|J \pi_{\ell, j}\right|_{\infty}+\operatorname{Lip}\left(J \pi_{\ell, j}\right)=D<\infty$. 
Proposition 4.2. Let $T$ be a piecewise $C^{1+\alpha}$ self-map of a metric space $(X, \tilde{d})$ with hole $\tilde{H}$. Suppose we can construct a Young tower over a reference set $\Lambda$ for which $T$ satisfies properties (A1) and (A2). Then $C^{\bar{\alpha}}(X) \subset \mathcal{P}_{\pi} \mathcal{B}_{0}$ for every $-\log \beta / \log \tau \leq \bar{\alpha} \leq \alpha$.

Proof. Let $\tilde{f} \in C^{\bar{\alpha}}(X)$ be given.

If $\pi\left(Z_{\ell, j}\right) \cap \pi\left(Z_{\ell^{\prime}, j^{\prime}}\right)=\emptyset$ for all other $\left(\ell^{\prime}, j^{\prime}\right) \in J$, then we can choose a single preimage for each $u \in \pi\left(Z_{\ell, j}\right)$ on which to define $f$. In fact, inverting the projection operator $\mathcal{P}_{\pi}$, we see that defining $f(x)=\tilde{f}(\pi x) J \pi(x)$ for each $x \in Z_{\ell, j}$ yields the correct value for $\tilde{f}(\pi x)$.

Now consider the case in which $\pi\left(Z_{\ell_{1}, j_{1}}\right) \cap \pi\left(Z_{\ell_{2}, j_{2}}\right) \neq \emptyset$. We may choose a partition of unity $\left\{\rho_{1}, \rho_{2}\right\}$ for $E=\pi\left(Z_{\ell_{1}, j_{1}} \cup Z_{\ell_{2}, j_{2}}\right)$ such that $\rho_{i} \in C^{\alpha}(E)$. Then we define $f$ by

$$
f_{\ell_{i}, j_{i}}\left(x_{i}\right)=\tilde{f}\left(\pi x_{i}\right) J \pi\left(x_{i}\right) \rho_{i}\left(\pi x_{i}\right)
$$

for $x_{i} \in Z_{\ell_{i}, j_{i}}$ and $i=1,2$. Then for $u \in E$, we set $f=0$ on preimages of $u$ which are not in $Z_{\ell_{1}, j_{1}} \cup Z_{\ell_{2}, j_{2}}$. It is clear that $\mathcal{P}_{\pi} f(u)=\tilde{f}(u)$ for $u \in E$.

This construction can be generalized to accommodate finitely many overlaps in the projections $\pi\left(Z_{\ell, j}\right)$ while maintaining a uniform bound on the $C^{\alpha}$-norm of the $\rho_{i}$.

Let $\mathcal{Z}_{J}=\bigcup_{(\ell, j) \in J} Z_{\ell, j}$. Lemma 4.1 tells us that $\tilde{f} \circ \pi \in \mathcal{B}_{0}$ (where $\mathcal{B}_{0}$ is defined in (2.3)) and (A2)(c) implies that $\left.J \pi\right|_{\mathcal{Z}_{J}} \in \mathcal{B}_{0}$. Since $f \equiv 0$ outside of $\mathcal{Z}_{J}$, it follows immediately that $f \in \mathcal{B}_{0}$.

4.2. Piecewise expanding maps of the interval. Proof of Theorem 2.10. Theorem A guarantees that $T$ admits a tower $(F, \Delta)$ satisfying properties (P1)-(P3) and (H1). Property (A1) is automatic for expanding maps.

It remains to verify that Property (A2) is satisfied. This follows from the tower construction contained in [D1]. For this class of maps, we may choose the reference set $\Lambda$ to be inside an interval of monotonicity of $T$ so that the finite partition of images $\mathcal{Z}_{0}^{i m}$ will consist of the single element $\Lambda$, i.e., we have a tower with full returns to the base. In the inductive construction of the partition $\mathcal{Z}_{0}$ on $\Lambda$, at each step, new pieces are created only by intersections with discontinuities, intersections with the hole, and returns to the base. In this way, only finitely many distinct pieces are generated by each iterate and therefore we have only finitely many overlaps when we project each level. Since $I$ is covered in finitely many iterates of $\Lambda$ by assumption (T1), it is also covered by the projection of finitely many levels of $\Delta$, say the first $N$. Thus if we take our index set $J$ to be all indices corresponding to elements in the first $N$ levels of the tower, it is immediate that (A2)(a) and (A2)(b) are satisfied.

To see that (A2)(c) is satisfied, let $x \in \Delta_{0}$ and notice that by (4.1)

$$
J \pi\left(F^{\ell} x\right)=J \pi(x) g_{\ell}(x) / \tilde{g}_{\ell}(\pi x) .
$$

If $\ell<R(x)$, then $J \pi(x)=g_{\ell}(x)=1$ so that

$$
J \pi\left(F^{\ell} x\right)=1 / \tilde{g}_{\ell}(\pi x)=\left|\left(T^{\ell}\right)^{\prime}(\pi x)\right| .
$$

Since $T$ is $C^{1+\alpha}$, so is $T^{\ell}$ for each $\ell$. Since we are only concerned with $\ell \leq N$ and $\bar{\alpha} \leq \alpha$, by Lemma $4.1,\left.J \pi\right|_{\mathcal{Z}_{J}} \in \mathcal{B}_{0}$ so (A2)(c) is satisfied. By Proposition 4.2, we have $C^{\alpha}(X) \subset \mathcal{P}_{\pi} B$. 
Property (T1) also implies that we can construct $(F, \Delta)$ to be mixing, since if $T^{n}\left(Z^{\prime}\right) \supseteq I$, then $T^{n+1}\left(Z^{\prime}\right) \supset I$ so we can avoid periodicity in the return time $R$ by simply delaying a return by one step. Applying Proposition 2.4, we see that $\mathcal{L}_{F}$ admits a unique probability density $\varphi$ for the eigenvalue $\lambda$ of maximum modulus. Defining $\tilde{\varphi}=\mathcal{P}_{\pi} \varphi$, we have $\mathcal{L}_{T}^{n} \tilde{f} /\left|\mathcal{L}_{T}^{n} \tilde{f}\right| \rightarrow \tilde{\varphi}$ at an exponential rate for every $\tilde{f} \in \mathcal{P}_{\pi} \mathcal{B}$ for which $c(\tilde{f})>0$ by Proposition 2.6.

Convergence property. Let $\tilde{f} \in \mathcal{G}$. Since $(F, \Delta)$ satisfies (A1) and (A2) and $\tilde{f} \in C^{\alpha}(I)$, by Proposition 4.2 we can find $f \in \mathcal{B}_{0}$, supported entirely in elements corresponding to the index set $J$, such that $\mathcal{P}_{\pi} f=\tilde{f}$. By Corollary 3.6, it suffices to show that $v(f)>0$, for then the convergence of $f$ to $\varphi$ will imply the convergence of $\tilde{f}$ to $\tilde{\varphi}:=\mathcal{P}_{\pi} \varphi$.

Since $\tilde{f} \in \mathcal{G}$, we have $\tilde{f}>0$ on $I^{\infty} \cap \Lambda$, which implies $f>0$ on $\Delta^{\infty} \cap \Delta_{0}$. Since $v$ is an invariant measure on $\Delta$, it must be that $v\left(\Delta_{0}\right)>0$ and so $v(f)>0$ as required.

Unified escape rate. Finally we prove that all functions in $\mathcal{G}$ have the same escape rate given by $-\log \lambda$. First note that given $\tilde{f} \in \mathcal{G}$ and $f \in \mathcal{B}$ such that $\mathcal{P}_{\pi} f=\tilde{f}$, we have

$$
\lim _{n \rightarrow \infty} \lambda^{-n} \mathcal{L}_{T}^{n} \tilde{f}=\lim _{n \rightarrow \infty} \lambda^{-n}\left|\mathcal{L}_{T}^{n} \tilde{f}\right|_{1} \frac{\mathcal{L}_{T}^{n} \tilde{f}}{\left|\mathcal{L}_{T}^{n} \tilde{f}\right|_{1}}=\lim _{n \rightarrow \infty} \lambda^{-n}\left|\mathcal{L}_{F}^{n} f\right|_{1} \frac{\mathcal{L}_{T}^{n} \tilde{f}}{\left|\mathcal{L}_{T}^{n} \tilde{f}\right|_{1}}=c(f) \tilde{\varphi}
$$

by Corollary 2.5 and the proof of convergence above. Since $v(f)>0$, we also have $c(f)>0$.

Thus if we let $\tilde{\eta}=\tilde{f} \tilde{m}$, we have

$$
\lim _{n \rightarrow \infty} \frac{1}{n} \log \tilde{\eta}\left(I^{n}\right)=\lim _{n \rightarrow \infty} \frac{1}{n} \log \left|\mathcal{L}_{T}^{n} \tilde{f}\right|_{1}=\log \lambda .
$$

4.3. Multimodal Collet-Eckmann maps with singularities. Proof of Theorem 2.12. The construction in [DHL] fixes $\delta$ and finds an interval $I^{*}$ with $c^{*} \in I^{*} \subset\left(c^{*}-\delta, c^{*}+\delta\right)$ as base for the induced map. We choose $I^{*}$ such that $\operatorname{orb}\left(\partial I^{*}\right)$ is disjoint from the interior of $I^{*}$. This is always possible by choosing $\partial I^{*}$ to be pre-periodic. Now by using $I^{*}$ as the base $\hat{\Delta}_{0}$ of the Young tower $\hat{\Delta}$ (i.e., without hole), and recalling that $\mathcal{Z}$ is the natural partition of the tower we have the following.

For any $Z, Z^{\prime} \in \mathcal{Z}$, the symmetric difference $\pi Z \Delta \pi Z^{\prime}=\emptyset$.

To show why this is true, write $Z=Z_{\ell, j}$ and $Z^{\prime}=Z_{\ell^{\prime}, j^{\prime}}$, so $\pi(Z)=T^{\ell}\left(\pi Z_{0, j}\right)$ and $\pi\left(Z^{\prime}\right)=T^{\ell^{\prime}}\left(\pi Z_{0, j^{\prime}}\right)$. Assume without loss of generality that $k:=R(Z)-\ell \geq R\left(Z^{\prime}\right)-$ $\ell^{\prime}=: k^{\prime}$. If (4.6) fails, then there are $x \in \partial \pi Z \cap \pi Z^{\prime}$ and $x^{\prime} \in \pi Z \cap \partial \pi Z^{\prime}$. In this case $\hat{T}^{k}\left(x^{\prime}\right)$ is an interior point of $I^{*}$, but at the same time $\hat{T}^{k}\left(x^{\prime}\right)=\hat{T}^{k-k^{\prime}}\left(\hat{T}^{k^{\prime}}\left(x^{\prime}\right)\right) \in$ $\hat{T}^{k-k^{\prime}}\left(\partial I^{*}\right)$. This contradicts the choice of $I^{*}$. We record property (4.6) for later use in checking condition (A2)(b).

Next we adapt the construction of the inducing for the system without hole from [DHL]. By (B2)(a) the artificial critical points $b_{j} \in \tilde{H}_{j}$ satisfy $\hat{T}^{k}\left(b_{j}\right) \neq c^{*}$ for all $k \geq 0$. Therefore (C3) still holds with the artificial critical points. We set the binding period of $x \in B_{\delta}\left(b_{j}\right)$ (see [DHL, §2.2]) to $p(x)=\tau(j)-1$ for $\tau(j)$ as in (2.5). Recall that the hole $\tilde{H}$ has $L$ components. When the image $\hat{T}^{n}(\omega)=\omega_{n}$ of a partition element $\omega$ visits $B_{\delta}\left(b_{j}\right)$ (see [DHL, p. 432]), we subdivide $\omega$ only if $\omega_{n}$ intersects $\partial \tilde{H}_{j}$. If $\omega_{n}$ has not escaped to 
large scale, so $\left|\omega_{n}\right|<\delta$, this results in at most three subintervals $\omega^{\prime} \subset \omega$ such that $\hat{T}^{n}\left(\omega^{\prime}\right)$ is either contained in $\tilde{H}_{j}$ or disjoint from $\tilde{H}$. By (2.5) and our choice of binding period $\left.p\right|_{B_{\delta}\left(b_{j}\right)}$, [DHL, Lemma 2] is automatically satisfied for $\theta^{*}:=\theta=\hat{\theta}=\lambda^{*}$.

Remark 4.3. In [DHL] close visits to $\mathrm{Crit}_{c} \cup \mathrm{Crit}_{s}$ that result in a cut are called essential returns, whereas those that do not result in a cut are called inessential returns. Let us call cuts caused by $\partial \tilde{H}$ hole returns. The cutting of $\omega^{\prime}$ at preimages of $\partial \tilde{H}_{j}$ is crucial for our tower to be compatible with the hole. Note also that by the slow recurrence condition (B1), a cutting of $\omega^{\prime}$ cannot occur within a binding period after a previous visit to a point in $\mathrm{Crit}_{c}$.

With this adaptation, the tower construction of [DHL] yields a tower $\hat{\Delta}$ and a return time function $\hat{R}$, constant on elements of the partition $\mathcal{Z}$. According to [DHL, Theorem 1], the tower $(\hat{F}, \hat{\Delta}, \hat{R})$ satisfies (P1)-(P3) and (A1) of the present paper.

Notice that at this point there is no escape. We have simply introduced new cuts at the boundaries of the hole during the construction of the return time function and partition of the interval $I^{*}$ so that the induced tower respects the boundary of the hole in the following sense: for each $Z \in \mathcal{Z}$, either $\pi Z \subset \tilde{H}$ or $\pi Z \cap \tilde{H}=\emptyset$.

A crucial feature of this construction is that the exponential rate $\theta$ of the tail behavior is independent of the size of $\tilde{H}$ when $\tilde{H}$ is small. To see this, recall the notation introduced in $\$ 2.4 .2$ regarding small holes. We first fix the set of points $b_{1}, \ldots b_{L}$, which we regard as infinitesimal holes satisfying (B1) and (B2). Then for each $h>0$, the family of holes $\mathcal{H}(h)$ consists of those holes $\tilde{H}$ satisfying: (1) $b_{j} \in \tilde{H}_{j}$ and $\tilde{m}\left(\tilde{H}_{j}\right) \leq h$ for each $1 \leq j \leq L$; and (2) $\tilde{H}$ satisfies (B1).

For the infinitesimal hole $\tilde{H}^{(0)}$ with components $\tilde{H}_{j}^{(0)}=b_{j}, j=1, \ldots, L$, we fix $\delta>0$ and a reference interval $I^{*} \subset B_{\delta}\left(c^{*}\right)$, and construct a tower $\Delta^{(0)}$ incorporating the additional cuts at $\partial \tilde{H}^{(0)}$ as described above.

An immediate concern is that the presence of additional cuts when we introduce holes of positive size interferes with returns to the extent that all full returns to $I^{*}$ are blocked. The following lemmas guarantee that this is not the case and in fact several properties such as mixing and the rate of returns persist for small holes.

LEMMA 4.4. For sufficiently small $h$, each $\tilde{H} \in \mathcal{H}(h)$ induces a tower $\hat{\Delta}^{(\tilde{H})}$ and return time function $\hat{R}^{(\tilde{H})}$ over $I^{*}$ using the construction described above. Moreover, $\left(\hat{F}^{(\tilde{H})}, \hat{\Delta}^{(\tilde{H})}\right)$ is mixing if $\left(\hat{F}^{(0)}, \hat{\Delta}^{(0)}\right)$ is mixing.

Proof. Notice that the thickening of the hole at the points $b_{j}$ cannot affect returns which happen before a fixed time $n_{h}$ depending only on $h$. For suppose $\omega \subset I^{*}$ satisfies $\hat{T}^{n} \omega=I^{*}$ where $n=\hat{R}^{(0)}(\omega) \leq n_{h}$ is the return time corresponding to $\tilde{H}^{(0)}$. Then in fact $\omega$ is in the middle third of a larger interval $\omega^{\prime}$ such that $\hat{T}^{n} \omega^{\prime} \supset I^{*}$. Cuts made by $\partial \tilde{H}_{j}$ must necessarily be at the endpoints of $\omega^{\prime}$ so for sufficiently small $h$, the return of $\omega$ will still take place at time $n$. By (B2)(a), we can force $n_{h} \rightarrow \infty$ as $h \rightarrow 0$, guaranteeing the persistence of returns up to any finite time for sufficiently small $h$.

To show $\left(\hat{F}^{(\tilde{H})}, \hat{\Delta}^{(\tilde{H})}\right)$ is mixing, we need only show that g.c.d. $\left(\hat{R}^{(\tilde{H})}\right)=1$ since $\hat{\Delta}^{(\tilde{H})}$ has a single base. Since $\left(\hat{F}^{(0)}, \hat{\Delta}^{(0)}\right)$ is mixing, there exists $N$ such that g.c.d. $\left\{\hat{R}^{(0)}: \hat{R}^{(0)} \leq\right.$ $N\}=1$. Now take $h$ small enough that $n_{h} \geq N$. Then g.c.d. $\left\{\hat{R}^{(\tilde{H})}\right\}=1$ as well. 
Our next lemma shows that the rate of return is uniform for small $h$.

LEMMA 4.5. There exist $\theta<1$ and $C>0$ such that $\tilde{m}\left(\hat{R}^{(\tilde{H})}>n\right) \leq C \theta^{n}$ for all $\tilde{H} \in$ $\mathcal{H}(h)$ with $h$ sufficiently small.

Proof. Let $\theta_{0}$ be the exponential rate of the tail behavior corresponding to $\tilde{H}^{(0)}$. We will show that by choosing $\delta$ and $h$ sufficiently small, we can make $\theta=\theta(\tilde{H})$ arbitrarily close to $\theta_{0}$ for all $\tilde{H} \in \mathcal{H}(h)$. We do this by showing that the rates of decay given by a series of lemmas in [DHL] vary little for small $h$.

Lemma 1 of [DHL]. Choose $h$ small enough that $n_{h}$ from the proof of Lemma 4.4 satisfies $n_{h} \geq t^{*}$ in Lemma 1. Then [DHL, Lemma 1] holds with the same rate since returns in the middle of large pieces are not affected by the hole before time $n_{h}$.

Lemma 6 of [DHL]. The notation $\mathcal{E}_{n, S}(\omega)$ stands for the set of subintervals within an interval $\omega$ of size $\delta / 3<|\omega|<\delta$ that have not grown to size $\delta$ by time $n$, and have essential return depths summing to $S$ within these $n$ iterates.

This lemma estimates the size of any interval $\omega^{\prime} \in \mathcal{E}_{n, S}$. Let us denote the number of hole returns used in the history of $\omega^{\prime}$ by $S_{\text {hole }}$. Define $\mathcal{E}_{n, S, S_{\text {hole }}}$ to be the set of subintervals $\omega^{\prime} \in \mathcal{E}_{n, S}$ such that $\omega^{\prime}$ has $S_{\text {hole }}$ hole returns in its history up to time $n$.

Every hole return, i.e., a cut at $\partial \tilde{H}_{j}$, is followed by a binding period of length $\tau(j)$ in which derivatives grow by an extra factor of 6 by (2.5). Since [DHL, Lemma 6] is concerned only with derivatives, and not with the actual cutting, the conclusion of [DHL, Lemma 6] becomes: for every $n \geq 1, S \geq 1$ and $S_{\text {hole }} \geq 1$ and $\omega^{\prime} \in \mathcal{E}_{n, S, S_{\text {hole }}}$ we have

$$
\left|\omega^{\prime}\right| \leq \kappa^{-1} e^{-\theta^{*} S} 6^{-S_{\text {hole }}}
$$

where $\theta^{*}$ replaces the $\theta$ used in [DHL, Lemma 2].

Lemma 7 of $[\mathbf{D H L}]$. This lemma relies on combinatorial estimates to obtain an upper bound on the number of pieces which can grow to size $\delta$ at specific times. By specifying the number of hole returns by $S_{\text {hole }}$ and using the fact that intervals are cut into at most three pieces during a hole return, we can adapt the conclusion of [DHL, Lemma 7] to

$$
\# \mathcal{E}_{n, S, S_{\text {hole }}}(\omega) \leq e^{\tilde{\eta} S_{3} S_{\text {hole }}} .
$$

Combining [DHL, Lemmas 6 and 7] in this form gives

$$
\begin{aligned}
\left|\mathcal{E}_{n, S}(\omega)\right| & =\sum_{S_{\text {hole }} \geq 0}\left|\mathcal{E}_{n, S, S_{\text {hole }}}(\omega)\right| \\
& \leq \sum_{S_{\text {hole }} \geq 0} \kappa^{-1} e^{-\theta^{*} S} 6^{-S_{\text {hole }}} e^{\tilde{\eta} S_{3} S_{\text {hole }}}=\kappa^{-1} e^{-\left(\theta^{*}-\tilde{\eta}\right) S}
\end{aligned}
$$

which is precisely [DHL, formula (21)].

The free time of an interval $\omega^{\prime}$ are all the iterates not spent in a binding period. We suppose that $\omega^{\prime}$ escapes to 'large scale' at time $n$ (i.e., $\left|\hat{T}^{n}\left(\omega^{\prime}\right)\right| \geq \delta$ ) and consider its history until time $n$. If $\omega^{\prime}$ is cut very short at a hole return, say $\partial \hat{T}^{m}\left(\omega^{\prime}\right) \cap \partial \tilde{H}_{j} \neq \emptyset$, then we first have a binding period of length $p(x)=\tau(j)-1$, and the free period after that lasts until either: (i) $\omega^{\prime}$ reaches large scale; (ii) $\omega^{\prime}$ has the next artificial cut near $b_{j} \in$ Crit hole $_{\text {; }}$ (iii) $\omega^{\prime}$ has an inessential return near $c \in \mathrm{Crit}_{c} \cup \mathrm{Crit}_{s}$; or (iv) $\omega^{\prime}$ has the next essential return 
near $c \in \mathrm{Crit}_{c} \cup \mathrm{Crit}_{s}$. In case (iv), $\hat{T}^{k}\left(\omega^{\prime}\right)$ covers at least three intervals in the exponential partition of $B_{\delta}(c)$ as in [DHL, p. 433].

Let us call the time from iterate $m+\tau(j)-1$ to the next occurrence of (i), (ii) or (iv) the extended free period of $\omega$ after iterate $m+\tau(j)-1$. Hence this includes binding and free periods after inessential returns to $B_{\delta}(c)$ for $c \in \mathrm{Crit}_{c} \cup \mathrm{Crit}_{s}$. These are the returns where $\hat{T}^{k}\left(\omega^{\prime}\right)$ is too short to result in a cut. Condition (B1) implies that when such an inessential return occurs, the next cut or inessential return will not occur until after the binding period associated to $\operatorname{dist}\left(\hat{T}^{k}\left(\omega^{\prime}\right), c\right)$. This binding period will restore the small derivative incurred at time $k$ due to [DHL, Lemma 2]. Hence there is $\lambda_{\text {hole }}$, depending only on $\lambda^{*}$ and $\Lambda^{*}$ from conditions (C1) and (C2), such that

$$
\left|D \hat{T}^{\ell}(x)\right| \geq e^{\lambda_{\text {hole } \ell}}
$$

for each $x \in \hat{T}^{m+\tau(j)-1}\left(\omega^{\prime}\right)$ and $\ell$ is the length of this extended free period. We let $n_{\text {hole }}$ denote the sum of extended free periods directly following the binding periods due to hole returns in the history of $\omega^{\prime}$ up until time $n$. With this notation, we make adaptations to the remaining lemmas.

Lemma 8 of [DHL]. This lemma can be changed to: there exists $n_{\delta}$ such that for every $\omega^{\prime} \in \mathcal{E}_{n, 0}$ with $S_{\text {hole }}=0, \omega^{\prime}=\omega$ and $n \leq n_{\delta}$. The reason is that intervals of definite size cannot remain small forever if they are not cut during an essential return or hole return, and in fact the $n_{\delta}$ can be taken equal to the $n(\delta)$ used in condition (B2).

Lemma 9 of [DHL]. This lemma can be restated as: for all $n \geq 1$ and $S \geq 0$ such that $\mathcal{E}_{n, S} \neq \emptyset$, we have

$$
S \geq\left(n-n_{\text {hole }}-n_{\delta}\right) / \tilde{\theta} .
$$

In other words, we disregard the hole free time $n_{\text {hole }}$. The proof is basically the same as in [DHL] if we keep in mind that at an essential return to $c \in \mathrm{Crit}_{c} \cup \mathrm{Crit}_{s}$ at time $\ell$, following a hole return, the size of the interval $\hat{T}^{\ell}\left(\omega^{\prime}\right)$ that emerges from the cut at this essential return depends only on the distance of $\hat{T}^{\ell}\left(\omega^{\prime}\right)$ to $c$.

Now [DHL, Lemmas 8 and 9] and (4.7) combine to give for $\mathcal{E}_{n}(\omega):=\bigcup_{S \geq 0} \mathcal{E}_{n, S}(\omega)$ :

$$
\begin{aligned}
\left|\mathcal{E}_{n}(\omega)\right| & =\sum_{0 \leq n_{\text {hole }} \leq n} \sum_{S \geq\left(n-n_{\text {hole }}-n_{\delta}\right) / \tilde{\theta}}\left|\mathcal{E}_{n, S,}(\omega)\right| \\
& \leq \sum_{0 \leq n_{\text {hole }} \leq n} e^{-\lambda_{\text {hole }} n_{\text {hole }}} \sum_{S \geq\left(n-n_{\text {hole }}-n_{\delta}\right) / \tilde{\theta}} \kappa^{-1} e^{-\left(\theta^{*}-\tilde{\eta}\right) S} \\
& \leq \tilde{C}_{1} \exp \left(\left(\theta^{*}-\tilde{\eta}\right) n_{\delta} / \tilde{\theta}\right) \exp \left(-\min \left\{\left(\theta^{*}-\tilde{\eta}\right) / \tilde{\theta}, \lambda_{\text {hole }}\right\} n\right),
\end{aligned}
$$

for some $\tilde{C}_{1}$ as in the formula given near the bottom of [DHL, p. 444]†.

Having established [DHL, Lemmas 6-9], the rest of the proof in [DHL] goes through basically unchanged since the decay in $\hat{R}^{(\tilde{H})}$ depends only on the rates in these lemmas and distortion estimates which are not affected by $\tilde{H}$. We see that $\theta(\tilde{H})$ can be made arbitrarily close to $\theta_{0}$ for $h$ sufficiently small.

We have shown that in the presence of additional cuts introduced by $\partial \tilde{H}$, we retain some uniform control over the induced towers $\left(\hat{F}^{(\tilde{H})}, \hat{\Delta}^{(\tilde{H})}\right)$. We are now ready to lift the holes into the towers and consider the open systems so defined.

$\dagger$ This is with the factor $\kappa^{-1}$ inserted where it is missing in [DHL]. 
We define the hole in the tower and the return time with hole to be

$$
H=\{Z \in \mathcal{Z}: \pi Z \subseteq \tilde{H}\} \quad \text { and } \quad R(x)=\min \left(\hat{R}(x), \min \left\{j: \hat{T}^{j}(x) \in \tilde{H}\right\}\right) .
$$

For any partition element $Z_{\ell, j}=H_{\ell, j}$ that is identified as a hole, we delete all levels in the tower above $Z_{\ell, j}$ since nothing is mapped to those elements once the hole is introduced. We denote the remaining tower with holes by $\Delta$ and define $F=\left.\hat{F}\right|_{\Delta \cap \hat{F}^{-1} \Delta}$ to be the corresponding tower map.

In order to invoke the conclusions of Proposition 2.6 for $(F, \Delta)$, we must check that its hypotheses, (P1)-(P3) and (H1), are satisfied. We then check conditions (A1) and (A2) in order to project the convergence results from the tower to the underlying system.

Properties (P1)-(P3) are automatic for $(F, \Delta, R)$ since they hold for $(\hat{F}, \hat{\Delta}, \hat{R})$.

Step 1. Condition (H1). We split the sum in (H1) into pieces that encounter $\tilde{H}$ during their bound period and those that encounter it when they are free,

$$
\sum_{\ell} m\left(H_{\ell}\right) \beta^{-\ell}=\sum_{\text {bound }} m\left(H_{\ell}\right) \beta^{-\ell}+\sum_{\text {free }} m\left(H_{\ell}\right) \beta^{-\ell} .
$$

To estimate the bound pieces, we use the slow recurrence condition given by (B1). If $\omega \subset I^{*}$ is some partition element, and $c \in \mathrm{Crit}_{c}$ the last critical point visited by $\omega$ before $\omega$ falls into the hole, then $\operatorname{dist}\left(\hat{T}^{\ell} c, \partial \tilde{H}_{j}\right) \geq \delta e^{-\alpha_{c}^{*} \ell}$ for each $j$. Therefore, if $\hat{T}^{\ell} \omega \cap \tilde{H}_{j} \neq \emptyset$, we must have $\delta e^{-\alpha_{c}^{*} \ell}<\tilde{m}\left(\tilde{H}_{j}\right)$ and so $\ell>-\left(1 / \alpha_{c}^{*}\right) \log \left(\tilde{m}\left(\tilde{H}_{j}\right) / \delta\right)$. Thus by Property (P1),

$$
\begin{aligned}
\sum_{\text {bound }} m\left(H_{\ell}\right) \beta^{-\ell} & \leq \sum_{\ell>-\left(1 / \alpha_{c}^{*}\right) \log (\tilde{m}(\tilde{H}) / \delta)} m\left(\hat{\Delta}_{\ell}\right) \beta^{-\ell} \\
& \leq \sum_{\ell>-\left(1 / \alpha_{c}^{*}\right) \log (\tilde{m}(\tilde{H}) / \delta)} C \theta^{\ell} \beta^{-\ell} \leq \frac{C^{\prime}}{\delta} \tilde{m}(\tilde{H})^{\left(1 / \alpha_{c}^{*}\right) \log \left(\theta^{-1} \beta\right) .}
\end{aligned}
$$

To estimate the free pieces, we will need some facts about the tower without holes, $(\hat{F}, \hat{\Delta})$. It was shown in $[\mathbf{Y}]$ that $\hat{F}$ admits a unique absolutely continuous invariant measure $\eta$ with density $\rho \in \mathcal{B}_{0}, \rho \geq a>0$. Moreover, $\pi_{*} \eta=\tilde{\eta}$ is the unique SRB measure for $\hat{T}$. By $\S 4.1, \tilde{\rho}=\mathcal{P}_{\pi} \rho$ is the density of $\tilde{\eta}$.

Notice that $\left.\rho\right|_{\hat{\Delta}_{0}}$ is an invariant density for $\hat{F}^{\hat{R}}$ so that $\tilde{\rho}_{0}:=\mathcal{P}_{\pi}\left(\rho_{\hat{\Delta}_{0}}\right)$ is an invariant density for $\hat{T}^{\hat{R}}$. Since $\pi^{\prime} \equiv 1$ on $\hat{\Delta}_{0}$, we have $a \leq \tilde{\rho}_{0} \leq A$. This implies that we can also obtain the invariant density $\tilde{\rho}$ by pushing forward $\tilde{\rho}_{0}$ under iterates of $\hat{T}$.

It is clear that pushing forward $\tilde{\rho}_{0}$ will result in spikes above the orbits of the critical points, hence $\tilde{\rho}$ is not bounded on $\hat{I}$. However, when an interval $\omega \subset \pi\left(\hat{\Delta}_{0}\right)$ is free at time $n$, condition (C2) and [DHL, Lemma 1] imply that the push forward of the density on $\omega$ at time $n$ will be uniformly bounded.

Define neighborhoods $N_{k}\left(\hat{T}^{k} c\right)$ of radius $\delta e^{-2 \alpha_{c}^{*} k}$ for each $c \in \mathrm{Crit}_{c}$. These are precisely the points starting in $B_{\delta}(c)$ whose orbits are still bound to $c$ at time $k$. From the above considerations, it is clear that outside of the set $\bigcup_{c \in \text { Crit }_{c}} \bigcup_{k \geq 1} N_{k}\left(\hat{T}^{k} c\right)$, the density $\tilde{\rho}$ is bounded. This is the sum of the push forwards of $\tilde{\rho}_{0}$ on free pieces. Thus, we may define a measure

$$
\tilde{\eta}_{\text {free }}=\sum_{(\ell, j): Z_{\ell, j} \text { is free }} \pi_{*} \eta\left(Z_{\ell, j}\right)
$$


whose density with respect to Lebesgue, $\tilde{\rho}_{\text {free }}$, is bounded on $\hat{I}$. Then since $\rho \geq a>0$, we have

$$
\sum_{\text {free }} m\left(H_{\ell, j}\right) \leq \sum_{\text {free }} \eta\left(H_{\ell, j}\right) / a=\tilde{\eta}_{\text {free }}(\tilde{H}) / a \leq C \tilde{m}(\tilde{H}) .
$$

Now set $P=-\log \tilde{m}(\tilde{H})$. We estimate the contribution from free pieces by

$$
\begin{aligned}
\sum_{\text {free }} m\left(H_{\ell, j}\right) \beta^{-\ell} & =\sum_{\text {free: } \ell>P} m\left(H_{\ell, j}\right) \beta^{-\ell}+\sum_{\text {free: } \ell \leq P} m\left(H_{\ell, j}\right) \beta^{-\ell} \\
& \leq \sum_{\text {free: } \ell>P} C \theta^{\ell} \beta^{-\ell}+\beta^{-P} \sum_{\text {free: } \ell \leq P} m\left(H_{\ell, j}\right) \\
& \leq C^{\prime}\left(\theta \beta^{-1}\right)^{P}+C^{\prime \prime} \beta^{-P} \tilde{m}(\tilde{H}) \\
& \leq C^{\prime} \tilde{m}(\tilde{H})^{\log \left(\beta \theta^{-1}\right)}+C^{\prime \prime} \tilde{m}(\tilde{H})^{1+\log \beta} .
\end{aligned}
$$

Putting together (4.9) and (4.10), we see that the left-hand side of (H1) is proportional to $\tilde{m}(\tilde{H})^{\gamma}$, for some $\gamma>0$. This quantity can be made sufficiently small to satisfy (H1) by choosing $\tilde{m}(\tilde{H})$ small since $\theta$ (and hence $\beta$ ) are independent of $\tilde{H}$ by Lemma 4.5.

Step 2. Property (A1). The bounded distortion required by (A1)(b) is satisfied by the cutting of pieces introduced in the construction of $\Delta$ (see [DHL, Proposition 3]). The expansion required by (A1)(a) follows from two estimates: property (C1) guarantees that starting at any $x \notin B_{\delta}(\mathrm{Crit})$, there is exponential expansion upon entry to $B_{\delta}(\mathrm{Crit})$; [DHL, Lemma 2] guarantees that exponential expansion occurs at the end of a binding period. Since any return must occur at a free entry to $B_{\delta}\left(c^{*}\right)$, we may concatenate these estimates as many times as needed in order to obtain (A1)(a) at any return time $\hat{R}_{n}$. However, once the hole is introduced, a partition element may fall into the hole during a bound period and so the return time with hole, $R$, may be declared when there has not been sufficient expansion to satisfy (A1)(a). Since this property is only needed to prove Lemma 4.1, we give an alternate proof of this lemma which uses (A1)(a) only for $\hat{R}$.

Proof of Lemma 4.1 for $(F, \Delta)$. First note that because (A1) is satisfied by $(\hat{F}, \hat{\Delta})$, Lemma 4.1 holds for lifts $\tilde{f} \circ \pi$ of $\tilde{f} \in C^{\bar{\alpha}}(\hat{I})$, with $\bar{\alpha} \geq-\log \beta / \log \tau$. Here $\tau$ is the rate of expansion from (A1) and $\beta$ is the constant chosen for the symbolic metric on $\hat{\Delta}$ (see $\$ 2.1 .1$ ).

The separation time $\hat{s}(\cdot, \cdot)$ is shortened by the introduction of the hole in the tower so that the new separation time satisfies $s(x, y) \leq \hat{s}(x, y)$. Thus the separation time metric is also loosened on $\Delta$ :

$$
d_{\beta}(x, y):=\beta^{s(x, y)} \geq \beta^{\hat{s}(x, y)}=: \hat{d}_{\beta}(x, y) .
$$

Thus if $f$ is Lipschitz with respect to $\hat{d}_{\beta}$ on $\hat{\Delta}$, its restriction to $\Delta$ is also Lipschitz with respect to $d_{\beta}$.

Now for $\tilde{f} \in C^{\bar{\alpha}}(\hat{I})$, with $\bar{\alpha} \geq-\log \beta / \log \tau$, we have $\tilde{f} \circ \pi \in \mathcal{B}_{0}(\hat{\Delta})$ by Lemma 4.1. Then by (4.11), $\tilde{f} \circ \pi \in \mathcal{B}_{0}(\Delta)$ as well.

Step 3. Property (A2). We focus first on finding an index set $J \subset \mathbb{N} \times \mathbb{N}$ such that (A2)(a) is satisfied. The following lemma is the analogue of (2.4) for $T$, the map with holes. (See also [D2, Lemma 5.2].) 
LEMMA 4.6. Let $\delta$ be the radius of $B_{\delta}\left(c^{*}\right)$ as above. Let $n_{0}=n(\delta)$ be defined by (2.4). For $h$ sufficiently small, given any interval $\omega \subset I$ such that $|\omega| \geq \delta / 3$, we have

$$
\bigcup_{i=0}^{2 n_{0}} T^{i} \omega \supset I \quad \bmod 0 .
$$

Proof. Suppose there exists an interval $A$ such that $A \cap\left(\bigcup_{i=0}^{n_{0}} T^{i} \omega\right)=\emptyset$. Since $A \subseteq$ $\hat{T}^{n_{0}} \omega$, we must have $A \cap \hat{T}^{i_{k}} \tilde{H}_{k} \neq \emptyset$ for some $\tilde{H}_{k}$ such that $\tilde{H}_{k} \cap \hat{T}^{i_{k}^{\prime}} \omega \neq \emptyset$ for some integers $i_{k}, i_{k}^{\prime}$ with $i_{k}+i_{k}^{\prime}=n_{0}$. In other words, the piece of $\omega$ that should have covered part of $A$ fell into $\tilde{H}_{k}$ before time $n_{0}$.

Condition (B2)(b) implies that there exists $1 \leq j_{k} \leq k$ such that

$$
\min _{1 \leq \ell \leq n_{0}} \operatorname{dist}\left(g_{k, j_{k}}, \hat{T}^{\ell} b_{k}\right)>0 .
$$

Thus, for small $h$, we have $\hat{T}^{\ell}\left(\tilde{H}_{k}\right) \cap B_{h}\left(g_{k, j_{k}}\right)=\emptyset$ for all $1 \leq \ell \leq n_{0}$. So $B_{h}\left(g_{k, i}\right)$ is covered by time $n_{0}$ under $T$, i.e., $B_{h}\left(g_{k, j_{k}}\right) \subset T^{n_{0}} \omega$.

Since $\hat{T}\left(b_{k}\right)=T\left(g_{k, j_{k}}\right)$, condition (B2)(a) says that $B_{h}\left(g_{k, j_{k}}\right)$ cannot fall into the hole before time $n_{0}$ for small $h$. Thus $T^{i_{k}} B_{h}\left(g_{k}, j_{k}\right) \supseteq \hat{T}^{i_{k}} \tilde{H}_{k}$ and we conclude that the part of $A$ which should have been covered by the piece of $\omega$ that fell into $\tilde{H}_{k}$ is at the latest covered at time $n_{0}+i_{k}$ by an interval passing through $B_{h}\left(g_{k, j_{k}}\right)$.

Doing this for each $k$, we have $A \subset \bigcup_{k=1}^{L} T^{i_{k}} B_{h}\left(g_{k, j_{k}}\right)$ and so $A \subset \bigcup_{i=0}^{2 n_{0}} T^{i} \omega$.

Lemma 4.6 implies that $I$ can be covered by the projection of finitely many levels of $\Delta$, say the first $N$. If $\pi\left(Z_{\ell, j}\right) \subset \pi\left(Z_{\ell^{\prime}, j^{\prime}}\right)$ and both $\ell, \ell^{\prime} \leq N$, we eliminate $(\ell, j)$ from our index set, but retain $\left(\ell^{\prime}, j^{\prime}\right)$. By (4.6), the remaining index set $J \subset\{0, \ldots, N-1\} \times \mathbb{N}$ satisfies (A2)(a) and (A2)(b). As before, set $\mathcal{Z}_{J}=\bigcup_{(\ell, j) \in J} Z_{\ell, j}$.

By (4.5), $J \pi\left(F^{\ell} x\right)=\left(T^{\ell}\right)^{\prime}(\pi x)$ so that we are only concerned with the first $N$ iterates of $T^{\ell}$. It is clear that if Crit ${ }_{s}=\emptyset$ and $T$ is globally $C^{2}$ then $\left.J \pi\right|_{\mathcal{Z}_{J}} \in \mathcal{B}_{0}$ by Lemma 4.1 and so (A2)(c) is satisfied.

In the case when Crit is non-empty, (A2)(c) does not hold and so Proposition 4.2 must be modified. We do this in Step 5 of the proof when we address the convergence property for the a.c.c.i.m.

Step 4. $(F, \Delta)$ is mixing. Since we have constructed a tower over a single base, it suffices to show that g.c.d. $(R)=1$. The fact that $\hat{T}$ is non-renormalizable guarantees that for the infinitesimal hole $\tilde{H}^{(0)},\left(\hat{F}^{(0)}, \Delta^{(0)}\right)$ can be constructed to be mixing by making g.c.d. $\left(\hat{R}^{(0)}\right)=1$. Indeed, (2.4) implies that as in the case of expanding maps, we can simply wait one time step on a given return to destroy any periodicity in $\hat{R}^{(0)}$. Once this is accomplished, Lemma 4.4 implies that $\left(\hat{F}^{(\tilde{H})}, \hat{\Delta}^{(\tilde{H})}\right)$ is mixing for $\tilde{H} \in \mathcal{H}(h)$ with $h$ small enough that g.c.d. $\left(\hat{R}^{(\tilde{H})}\right)$ is still one (by making $n_{h}$ sufficiently large). But since holes cannot affect returns before level $n_{h}$ in $\Delta$, the tower with holes, we have that g.c.d. $(R)=1$ as well.

Step 5. Convergence property. We have already verified in Steps 2 and 3 that $(F, \Delta)$ satisfies (A1) and there is an index set $J$ satisfying (A2)(a) and (A2)(b).

By (4.5), the problem spots where $\left(T^{\ell}\right)^{\prime}$ (and therefore $J \pi$ ) are unbounded are neighborhoods of $T^{k}(c)$ for $c \in \mathrm{Crit}_{s}, k \geq 1$. In fact, we only need to address the iterates of 
$c \in \mathrm{Crit}_{s}$ up until the time when a neighborhood of $T^{k}(c)$ is covered by some other element in the tower on which the derivative is bounded. Since $I$ is covered by the first $N$ levels of $\Delta$, we need consider at most the first $N$ iterates of $c \in \mathrm{Crit}_{s}$.

Notice that if a neighborhood $A$ of $T^{k}(c)$ can only be reached by an interval $\omega$ originating in a neighborhood of $c$, then owing to the exponential partition of $B_{\delta}(c)$ which subdivides $\omega$, there are countably many elements $Z \subset \Delta_{\ell}$ whose projections cover $A$ and in which $\left|\pi^{\prime}\right|$ becomes unbounded the closer that $\pi Z$ is to $T^{k}(c)$.

Fix $\varepsilon>0$ and let $\mathcal{N}_{\varepsilon}(c)$ denote the $\varepsilon$-neighborhood of those iterates of $c \in \mathrm{Crit}_{s}$ which can only be reached by passing through $B_{\delta}(c)$. Let $\mathcal{N}_{\varepsilon}=\bigcup_{c \in \text { Crit }_{s}} \mathcal{N}_{\varepsilon}(c)$ and let $J_{1} \subset J$ be the index set of those elements $Z$ such that $\pi Z \subset \mathcal{N}_{\varepsilon}$. Denote by $1_{\varepsilon}$ the indicator function of the set $\left\{y \in I: y \in \pi Z_{\ell, j},(\ell, j) \in J_{1}\right\}$.

Now let $\tilde{f} \in \mathcal{G}$ and write $\tilde{f}=\tilde{f}_{0}+\tilde{f}_{\varepsilon}$ where $\tilde{f}_{\varepsilon}:=\tilde{f} \cdot 1_{\varepsilon}$ and $\tilde{f}_{0}=\tilde{f}-\tilde{f}_{\varepsilon}$. We define a lift of $\tilde{f}$ by $f=\tilde{f} \circ \pi \cdot J \pi$ on elements of $J$ as in the proof of Proposition 4.2. The lifts $f_{0}$ and $f_{\varepsilon}$ are defined analogously. Although $f \notin \mathcal{B}$, we do have $f_{0} \in \mathcal{B}_{0}$ by Proposition 4.2 since $\tilde{f}_{0} \circ \pi \equiv 0$ on those elements in which $J \pi$ becomes unbounded. Using Corollary 3.6 precisely as in $\S 4.2$, we have

$$
\lim _{n \rightarrow \infty} \lambda^{-n} \mathcal{L}^{n} \tilde{f}_{0}=c\left(\tilde{f}_{0}\right) \tilde{\varphi}
$$

where convergence is in the $L^{1}$-norm and $c\left(\tilde{f}_{0}\right)>0$. Since

$$
\lambda^{-n} \mathcal{L}^{n} \tilde{f}=\lambda^{-n} \mathcal{L}^{n} \tilde{f}_{0}+\lambda^{-n} \mathcal{L}^{n} \tilde{f}_{\varepsilon}
$$

our strategy will be to show that the $L^{1}$-norm of the second term above can be made uniformly small in $n$ by making $\varepsilon$ small. This will imply that $\lambda^{-n} \mathcal{L}^{n} \tilde{f} \rightarrow c(\tilde{f}) \tilde{\varphi}$ in $L^{1}(\tilde{m})$ where $c(\tilde{f})=\lim _{\varepsilon \rightarrow 0} c\left(\tilde{f}_{0}\right)>0$, implying the desired convergence result.

Estimating $\left|\mathcal{L}^{n} \tilde{f}_{\varepsilon}\right|_{L^{1}(\tilde{m})}$ is equivalent to estimating $\left|\mathcal{L}^{n} f_{\varepsilon}\right|_{L^{1}(m)}$.

$$
\begin{aligned}
\lambda^{-n} \int \mathcal{L}^{n} f_{\varepsilon} d m & =\lambda^{-n} \sum_{(\ell, j) \in J_{1}} \int_{\Delta^{n} \cap Z_{\ell, j}} f_{\varepsilon} d m \\
& \leq \lambda^{-n} \sum_{(\ell, j) \in J_{1}}|\tilde{f}|_{\infty}\left|J \pi_{\ell, j}\right|_{\infty} m\left(\Delta^{n} \cap Z_{\ell, j}\right) .
\end{aligned}
$$

Since we are concerned with finitely many problem spots where the derivative blows up, it suffices to show that the sum in (4.13) over elements in $J_{1}$ corresponding to one of the problem spots is proportional to $\varepsilon$. For simplicity, we fix $c \in \mathrm{Crit}_{s}$ and denote by $\mathcal{A}_{\varepsilon}$ the set of elements in $\Delta$ projecting to the $\varepsilon$-neighborhood of $\hat{T}(c)$. There exists $k_{\varepsilon}>0$ such that if $Z \in \mathcal{A}_{\varepsilon}$, then $\pi Z$ lies in an element of the partition $E_{k}^{-}=\hat{T}\left(c-e^{-k+1}, c-e^{-k}\right)$ and $E_{k}^{+}=\hat{T}\left(c+e^{-k}, c+e^{-k+1}\right)$ with $k \geq k_{\varepsilon}$.

For $Z_{\ell, j} \in \mathcal{A}_{\varepsilon}$, let $Z_{0, j}=F^{-\ell} Z_{\ell, j}$. We split the sum in (4.13) into those elements $Z \in \mathcal{A}_{\varepsilon}$ with $R(Z) \geq n$ and those with $R(Z)<n$. Let $0<\ell_{c}<1$ denote the critical order of $c$. 
We estimate terms with $R(Z)<n$ using Corollary 3.5 and the bounded distortion given by (A1)(b) for $J \pi$.

$$
\begin{aligned}
& \lambda^{-n} \sum_{Z_{\ell, j} \in \mathcal{A}_{\varepsilon}: R\left(Z_{\ell, j}\right)<n}\left|J \pi_{\ell, j}\right|_{\infty} m\left(\Delta^{n} \cap Z_{\ell, j}\right) \\
& \quad \leq \sum_{Z_{\ell, j} \in \mathcal{A}_{\varepsilon}: R\left(Z_{\ell, j}\right)<n}\left|J \pi_{\ell, j}\right|_{\infty} C \lambda^{-R\left(Z_{\ell, j}\right)} m\left(Z_{\ell, j}\right) \leq C^{\prime} \int_{\mathcal{A}_{\varepsilon}: R(z)<n} J \pi \lambda^{-R} d m \\
& \quad \leq C^{\prime}\left(\int_{\mathcal{A}_{\varepsilon}: R(Z)<n}(J \pi)^{p} d m\right)^{1 / p}\left(\int_{\mathcal{A}_{\varepsilon}: R(Z)<n} \lambda^{-R p /(p-1)} d m\right)^{1-1 / p}
\end{aligned}
$$

where $1<p<1 /\left(1-\ell_{c}\right)$. By (4.5), we have $J \pi_{\ell, j}=\left|\left(T^{\ell}\right)^{\prime}\right|_{Z_{0, j}} \mid$ so that if $\pi\left(Z_{\ell, j}\right) \subset$ $E_{k}^{ \pm}$, we have $J \pi_{\ell, j} \approx e^{k\left(1-\ell_{c}\right)}$. Also, $J \pi_{\ell, j}$ has bounded distortion across all $Z_{\ell, j}$ that project into a single $E_{k}^{ \pm}$. So we estimate the first factor in (4.14) by

$$
\begin{aligned}
\sum_{Z_{\ell, j} \in \mathcal{A}_{\varepsilon}: R(Z)<n}\left|J \pi_{\ell, j}\right|_{\infty}^{p} m\left(Z_{\ell, j}\right) & \leq \sum_{k \geq k_{\varepsilon}} \sum_{\pi} C \subset E_{k}^{ \pm} \\
& \leq \sum_{k \geq k_{\varepsilon}} C \exp \left(k\left(1-\ell_{c}\right) p\right) m\left(Z_{0, j}\right) \\
& \left.\leq C \exp \left(-k_{\varepsilon}\left(1-p\left(1-\ell_{c}\right) p\right) \exp (-k)\right)\right)
\end{aligned}
$$

To estimate the second factor in (4.14), notice that if $\pi Z \subset E_{k}^{ \pm}$, then $R(Z)>\log k$. Thus

$$
\begin{aligned}
\sum_{Z_{\ell, j} \in \mathcal{A}_{\varepsilon}: R(Z)<n} \lambda^{-R(Z) p /(p-1)} m(Z) & \leq \sum_{r>\log k_{\varepsilon}} \sum_{R(Z)=r} \lambda^{-r p /(p-1)} m(Z) \\
& \leq C \sum_{r>\log k_{\varepsilon}}\left(\lambda^{-p /(p-1)} \theta\right)^{r} \leq C^{\prime}\left(\lambda^{-p /(p-1)} \theta\right)^{\log k_{\varepsilon}} .
\end{aligned}
$$

To estimate the terms of (4.13) with $R(Z) \geq n$, notice that, for such $Z, \Delta^{n} \cap Z=Z$.

$$
\begin{aligned}
\lambda^{-n} \sum_{Z \in \mathcal{A}_{\varepsilon}: R(Z) \geq n}\left|J \pi_{\ell, j}\right|_{\infty} m(Z) & \leq C \lambda^{-n} \sum_{k \geq k_{\varepsilon}} e^{k\left(1-\ell_{c}\right)} \sum_{\pi Z \subset E_{k}^{ \pm}: R(Z) \geq n} m(Z) \\
& \leq C \lambda^{-n} \sum_{k \geq k_{\varepsilon}} e^{k\left(1-\ell_{c}\right)} m\left(\mathcal{A}_{\varepsilon} \cap \pi^{-1} E_{k}^{ \pm} \cap\{R \geq n\}\right) .
\end{aligned}
$$

We let $A_{k, n}=\mathcal{A}_{\varepsilon} \cap \pi^{-1} E_{k}^{ \pm} \cap\{R \geq n\}$. On the one hand, since $R>n$ on $A_{k, n}$, we have $m\left(A_{k, n}\right) \leq C \theta^{n}$; on the other hand, $m\left(A_{k, n}\right) \leq e^{-k}$ by definition of the partition. Choose $0<\gamma<\ell_{c}$ and write $m\left(A_{k, n}\right)=m\left(A_{k, n}\right)^{\gamma} m\left(A_{k, n}\right)^{1-\gamma}$. Then

$$
\begin{aligned}
\lambda^{-n} \sum_{Z \in \mathcal{A}_{\varepsilon}: R \geq n}\left|J \pi_{\ell, j}\right|_{\infty} m(Z) & \leq C \lambda^{-n} \sum_{k \geq k_{\varepsilon}} e^{k\left(1-\ell_{c}\right)} \theta^{n \gamma} e^{-k(1-\gamma)} \\
& \leq C^{\prime}\left(\lambda^{-1} \theta^{\gamma}\right)^{n} e^{-k_{\varepsilon}\left(\ell_{c}-\gamma\right)} .
\end{aligned}
$$

Putting together (4.15), (4.16) and (4.17) we see that (4.13) becomes

$$
\begin{aligned}
\lambda^{-n} \int \mathcal{L}^{n} f_{\varepsilon} d m \leq & C \exp \left(-k_{\varepsilon}\left(1-p\left(1-\ell_{c}\right)\right) / p\right)\left(\lambda^{-1} \theta^{(p-1) / p}\right)^{\log k_{\varepsilon}} \\
& +C^{\prime}\left(\lambda^{-1} \theta^{\gamma}\right)^{n} \exp \left(-k_{\varepsilon}\left(\ell_{c}-\gamma\right)\right) .
\end{aligned}
$$


When the holes are sufficiently small, i.e., when $\lambda^{-1} \geq \max \left\{\theta^{\gamma}, \theta^{(p-1) / p}\right\}$, this quantity can be made arbitrarily small independently of $n$.

Step 6. Exponential rate of convergence. We show that the convergence of $\lambda^{-n} \mathcal{L}^{n} \tilde{f}$ established in Step 5 occurs at an exponential rate. Since $\left|\mathcal{L}^{n} \tilde{f}\right|_{L^{1}(\tilde{m})}=\left|\mathcal{L}^{n} f\right|_{L^{1}(m)}$, it suffices to show this convergence for the lift on $\Delta$.

Let $\varepsilon=e^{-t n}$ for some small constant $t$ to be chosen later. Define $\mathcal{N}_{\varepsilon}$ as above and notice that outside of $\mathcal{N}_{\varepsilon}$, the $C^{2}$ norm of $\hat{T}^{\ell}$ for $\ell=1, \ldots, N$ is proportional to $e^{-k_{\varepsilon}\left(\ell_{c}^{*}-2\right)}$ where $\ell_{c}^{\min }>0$ is the minimum of the critical orders of $c \in \mathrm{Crit}_{s}$. Since $k_{\varepsilon}$ is on the order of $-\log \varepsilon$, we have $\left.\left|\hat{T}^{\ell}\right|_{\hat{I} \backslash \mathcal{N}_{\varepsilon}}\right|_{C^{2}}=\mathcal{O}\left(e^{t n\left(2-\ell_{c}^{\min }\right)}\right)$. Let $\mathcal{Z}_{J}=\bigcup_{(\ell, j) \in J} Z_{\ell, j}$ and let $\mathcal{Z}_{J, \varepsilon} \subset \mathcal{Z}_{J}$ denote those elements which project into $\mathcal{N}_{\varepsilon}$. Then

$$
\left\|J \pi \mid \mathcal{Z}_{J} \backslash \mathcal{Z}_{J, \varepsilon}\right\|_{0} \leq C e^{\operatorname{tn}\left(2-\ell_{c}^{\min }\right)} .
$$

Define $\tilde{f}_{0}, f_{0}, \tilde{f}_{\varepsilon}, f_{\varepsilon}$ as in Step 5. By Lemma 4.1, (4.19) implies that $\left\|f_{0}\right\| \leq C e^{\operatorname{tn}\left(2-\ell_{c}^{\min }\right)}$ so that by Corollary 2.5,

$$
\left|\lambda^{-n} \mathcal{L}^{n} f_{0}-c\left(f_{0}\right) \varphi\right|_{L^{1}(m)} \leq\left\|\lambda^{-n} \mathcal{L}^{n} f_{0}-c\left(f_{0}\right) \varphi\right\| \leq C e^{t n\left(2-\ell_{c}^{\min }\right)} \sigma^{n} .
$$

Next, when the holes are sufficiently small, $\lambda^{-1} \geq \max \left\{\theta^{\gamma}, \theta^{(p-1) / p}\right\}$, so (4.18) yields,

$$
\begin{aligned}
\lambda^{-n}\left|\mathcal{L}^{n} f_{\varepsilon}\right|_{1} & \leq C \exp \left(-\operatorname{tn}\left(1-p\left(1-\ell_{c}^{\min }\right)\right) / p\right)+C^{\prime} \exp \left(-\operatorname{tn}\left(\ell_{c}^{\min }-\gamma\right)\right) \\
& \leq C^{\prime \prime} \exp \left(-t n \gamma^{\prime}\right)
\end{aligned}
$$

for some $\gamma^{\prime}>0$. In particular, we see from (4.21) that the constants $c\left(f_{0}\right)$ converge to $c(f)$ exponentially fast as well

$$
\left|c(f)-c\left(f_{0}\right)\right|=\lim _{n \rightarrow \infty} \lambda^{-n}\left(\left|\mathcal{L}^{n} f\right|_{1}-\left|\mathcal{L}^{n} f_{0}\right|_{1}\right)=\lim _{n \rightarrow \infty} \lambda^{-n} \int \mathcal{L}^{n} f_{\varepsilon} d m \leq C^{\prime \prime} e^{-t n \gamma^{\prime}} .
$$

This estimate together with (4.20) and (4.21) imply that $\lambda^{-n} \mathcal{L} f \rightarrow c(f) \varphi$ exponentially fast once we choose $t<-\log \sigma /\left(2-\ell_{c}^{\min }\right)$.

Step 7. Unified escape rate. By Step 5, for each $\tilde{f} \in \mathcal{G}$, we have $f \in L^{1}(m)$ such that $\mathcal{P}_{\pi} f=\tilde{f}$ and $\lambda^{-n} \mathcal{L}_{F}^{n} f=c(f) \varphi$ for some $c(f)>0$ which implies $\lambda^{-n} \mathcal{L}_{T}^{n} \tilde{f}=c(f) \tilde{\varphi}$ by (4.3). Letting $\tilde{\eta}=\tilde{f} \tilde{m}$, we have

$$
\lim _{n \rightarrow \infty} \frac{1}{n} \log \tilde{\eta}\left(I^{n}\right)=\lim _{n \rightarrow \infty} \frac{1}{n} \log \left|\mathcal{L}_{T}^{n} \tilde{f}\right|_{1}=\log \lambda .
$$

4.3.1. Small hole limit. Proof of Theorem 2.14. By (4.9) and (4.10), the quantity $q:=\sum_{\ell \geq 1} m\left(H_{\ell}\right) \beta^{-(\ell-1)}$ can be made arbitrarily small by choosing $h$ to be small. By Proposition 3.1, the escape rate $\lambda$ is controlled by the size of $q$ so that $\lambda \rightarrow 1$ as $q \rightarrow 0$. Thus $\lambda_{h} \rightarrow 1$ as $h \rightarrow 0$.

Since $\tilde{\mu}_{h}$ is a sequence of probability measures on the compact interval $\hat{I}$, it follows that a subsequence, $\left\{\tilde{\mu}_{k}\right\}$ corresponding to $h_{k}$, converges weakly to a probability measure $\tilde{\mu}_{\infty}$. We show that $\tilde{\mu}_{\infty}$ is an absolutely continuous invariant measure for $\hat{T}$. Since there is only one such measure, this will imply that in fact the entire sequence converges to this same invariant measure. 
Step 1. $\tilde{\mu}_{\infty}$ is absolutely continuous with respect to Lebesgue. For each $\tilde{H}^{(k)}$, we have two towers: $\left(\hat{F}^{(k)}, \hat{\Delta}^{(k)}\right)$ which has no holes but is constructed using $\partial \tilde{H}^{(k)}$ as artificial cuts as described in the proof of Theorem 2.12; and $\left(F^{(k)}, \Delta^{(k)}\right)$, the tower with holes obtained from $\hat{\Delta}^{(k)}$. By Lemma 4.5, there exist uniform constants $C>0, \theta<1$ such that $m\left(\hat{\Delta}_{\ell}^{(k)}\right) \leq C \theta^{\ell}$.

We have an invariant density $\rho_{k}$ on $\hat{\Delta}^{(k)}$ and a conditionally invariant density $\varphi_{k}$ on $\Delta^{(k)}$. By Proposition 3.1, both $\rho_{k}, \varphi_{k} \in \mathcal{B}_{M}$ where $M$ is independent of $k$ (to see the results for $\rho_{k}$, simply apply the proposition to the case $H=\emptyset$ ). In addition, by Proposition 2.4(i), $\rho_{k} \geq a>0$ and the constant $a$ is independent of $k$ because the uniform decay given by Lemma 4.5 implies that $\hat{\Delta}_{0}^{(k)}$ must retain some positive minimum measure for all $k$.

Let $\hat{\pi}_{k}$ be the projection corresponding to $\hat{\Delta}^{(k)}$ and let $\pi_{k}=\left.\hat{\pi}_{k}\right|_{\Delta^{(k)}}$. Letting $\tilde{\rho}$ denote the unique invariant density for $\hat{T}$ and $J \hat{\pi}_{k}$ the Jacobian of $\hat{\pi}_{k}$ etc, we have for each $k$,

$$
\tilde{\rho}(x)=\mathcal{P}_{\hat{\pi}_{k}} \rho_{k}(x)=\sum_{y \in \hat{\pi}_{k}^{-1} x} \frac{\rho_{k}(y)}{J \hat{\pi}_{k}(y)} \quad \text { and } \quad \tilde{\varphi}_{k}(x):=\mathcal{P}_{\pi_{k}} \varphi_{k}(x) \sum_{y \in \pi_{k}^{-1} x} \frac{\varphi_{k}(y)}{J \pi_{k}(y)} .
$$

Now for any $\varepsilon>0$, choose $L>0$ such that $\sum_{\ell>L} C M \beta^{-\ell} \theta^{\ell}<\varepsilon$. Next choose $k_{0}$ such that for all $k \geq k_{0}, \lambda_{k}^{-L} \leq 2$. Now for any Borel $A \subset \hat{I}$,

$$
\tilde{\mu}_{k}(A)=\sum_{\ell \leq L} \mu_{k}\left(\Delta_{\ell} \cap \pi_{k}^{-1} A\right)+\sum_{\ell>L} \mu_{k}\left(\Delta_{\ell} \cap \pi_{k}^{-1} A\right)=: \tilde{\mu}_{k, L}(A)+\tilde{\mu}_{k,+}(A) .
$$

By (4.22), the measure $\tilde{\mu}_{k, L}$ has density $\tilde{\varphi}_{k, L}$ bounded independently of $k \geq k_{0}$ :

$$
\tilde{\varphi}_{k, L}(x)=\sum_{y \in \pi_{k}^{-1} x: \ell(y) \leq L} \frac{\varphi_{k}(y)}{J \pi_{k}(y)} \leq \frac{M}{a} \sum_{y \in \hat{\pi}_{k}^{-1} x: \ell(y) \leq L} \lambda_{k}^{-\ell(y)} \frac{\rho_{k}(y)}{J \hat{\pi}_{k}^{-1}(y)} \leq \frac{2 M \tilde{\rho}(x)}{a}
$$

where $\ell(y)$ is the level of $y$ in $\hat{\Delta}^{(k)}$. The remaining measure $\tilde{\mu}_{k,+}$ has small total mass:

$$
\tilde{\mu}_{k,+}(\hat{I})=\sum_{\ell>L} \tilde{\mu}_{k}\left(\Delta_{\ell}\right) \leq \sum_{\ell>L} M \beta^{-\ell} m\left(\Delta_{\ell}\right) \leq \sum_{\ell>L} C M \beta^{-\ell} \theta^{\ell}<\varepsilon .
$$

Putting together (4.23) and (4.24), we see that $\mu_{\infty}=\mu_{\infty, L}+\mu_{\infty,+}$ where $\mu_{\infty, L}$ has density bounded by $2 M \tilde{\rho} / a$ while $\mu_{\infty,+}$ is possibly singular with total mass less than $\varepsilon$. Since this is true for each $\varepsilon>0$, we conclude that in fact $\mu_{\infty}$ is absolutely continuous with density bounded by $2 M \tilde{\rho} / a$.

Step 2. $\tilde{\mu}_{\infty}$ is invariant. Let $I_{k}=\hat{I} \backslash \tilde{H}^{(k)}$ and, as usual, let $I_{k}^{n}=\bigcap_{j=0}^{n} \hat{T}^{-j} I_{k}$ and $T_{k}=\left.\hat{T}\right|_{I_{k}^{1}}$.

By Step $1, \tilde{\mu}_{\infty}$ has density bounded by $2 M \tilde{\rho} / a$, which is in $L^{1}(\tilde{m})$. Thus $\tilde{\mu}_{\infty}$ gives zero measure to the singularity points of $\hat{T}$. This fact allows us to write, for any continuous function $f$ on $\hat{I}$,

$$
\tilde{\mu}_{\infty}(f \circ \hat{T})=\lim _{k \rightarrow \infty} \tilde{\mu}_{k}(f \circ \hat{T})=\lim _{k \rightarrow \infty} \int_{I_{k}^{1}} f \circ T_{k} d \tilde{\mu}_{k}+\int_{\hat{I} \backslash I_{k}^{1}} f \circ \hat{T} d \tilde{\mu}_{k} .
$$

Since $\lambda_{k} \rightarrow 1$, the first term in (4.25) is equal to

$$
\lim _{k \rightarrow \infty} \int_{I_{k}} f d\left(\left(T_{k}\right)_{*} \tilde{\mu}_{k}\right)=\lim _{k \rightarrow \infty} \lambda_{k} \tilde{\mu}_{k}(f)=\tilde{\mu}_{\infty}(f) .
$$

The second term in (4.25) is bounded by $|f|_{\infty} \tilde{\mu}_{k}\left(\hat{I} \backslash I_{k}^{1}\right)$. This quantity tends to zero as $k \rightarrow \infty$ because of the uniform bounds on the densities of $\tilde{\mu}_{k}$ obtained in Step 1 . 


\section{Equilibrium principle}

In this section we consider the invariant measures $v$ and $\tilde{v}=\pi_{*} v$ and prove Theorems 2.9, 2.16 and 2.17. We assume throughout that $F$ is mixing and satisfies (P1)-(P3) and (H1).

5.1. Characterization of $\tilde{v}$. Proof of Theorem 2.16. Let $\tilde{f} \in C^{\bar{\alpha}}(X)$ and note that $\tilde{f} \circ \pi \in \mathcal{B}_{0}$. Thus,

$$
\begin{aligned}
\tilde{v}(\tilde{f}) & =v(\tilde{f} \circ \pi)=\lim _{n \rightarrow \infty} \lambda^{-n} \int_{\Delta^{n}} \tilde{f} \circ \pi d \mu \\
& =\lim _{n \rightarrow \infty} \lambda^{-n} \int_{\Delta^{n}} \tilde{f} \circ \pi \varphi d m=\lim _{n \rightarrow \infty} \lambda^{-n} \int_{\pi\left(\Delta^{n}\right)} \mathcal{P}_{\pi}(\tilde{f} \circ \pi \varphi) d \tilde{m} \\
& =\lim _{n \rightarrow \infty} \lambda^{-n} \int_{X^{n}} \tilde{f} \tilde{\varphi} d \tilde{m}=\lim _{n \rightarrow \infty} \lambda^{-n} \int_{X^{n}} \tilde{f} d \tilde{\mu}
\end{aligned}
$$

where in the first line we have used Proposition 2.8.

The ergodicity of $\tilde{v}$ follows from that of $v$ and the relation $X^{\infty}=\pi\left(\Delta^{\infty}\right)$. If $A \subset X$ is $T$-invariant, then since $F^{-1} \circ \pi^{-1}(A)=\pi^{-1} \circ T^{-1}(A)=\pi^{-1}(A)$, we conclude that $\pi^{-1}(A)$ is $F$-invariant. This implies that $\tilde{v}(A)$ is 0 or 1 .

To prove exponential decay of correlations let $\tilde{f}_{1}, \tilde{f}_{2} \in C^{\alpha}(X)$. Set $f_{i}=\tilde{f}_{i} \circ \pi$ and note that $\int_{X} \tilde{f}_{i} d \tilde{v}=\int_{\Delta} \tilde{f}_{i} \circ \pi d \nu$ for $i=1,2$. So

$$
\begin{aligned}
\int_{X} \tilde{f}_{1} \tilde{f}_{2} \circ T^{n} d \tilde{v} & =\int_{\Delta} \tilde{f}_{1} \circ \pi \tilde{f}_{2} \circ T^{n} \circ \pi d v \\
& =\int_{\Delta} f_{1} \tilde{f}_{2} \circ \pi \circ F^{n} d v=\int_{\Delta} f_{1} f_{2} \circ F^{n} d v
\end{aligned}
$$

from which exponential decay of correlations follows using Proposition 2.8 and the fact that $f_{1}, f_{2} \in \mathcal{B}_{0}$.

5.2. Equilibrium principle on the tower. First note that since $F$ is mixing, Property (P3) implies that there exists an $n_{0} \in \mathbb{N}$ such that $F^{n}\left(Z^{\prime}\right) \supseteq \Delta_{0}$, for all $n \geq n_{0}$ and $Z^{\prime} \in \mathcal{Z}_{0}^{\text {im }}$.

Let $v_{0}:=\left.\left(1 / v\left(\Delta_{0}\right)\right) v\right|_{\Delta_{0}}$ and define $S=F^{R}: \Delta^{\infty} \cap \Delta_{0} \circlearrowleft$. Let $R_{n}(x)=\sum_{k=0}^{n-1} R\left(S^{k} x\right)$ be the $n$th return time starting at $x$ and let $\mathcal{M}_{S}$ be the set of $S$-invariant Borel probability measures on $\Delta^{\infty} \cap \Delta_{0}$.

PROPOSITION 5.1. The measure $v_{0}$ is a Gibbs measure for $S$ and $S$ is topologically mixing on $\Delta^{\infty}$. Accordingly,

$$
\sup _{\eta_{0} \in \mathcal{M}_{S}}\left\{h_{\eta_{0}}(S)+\int_{\Delta_{0}} \log \left((J S)^{-1} \lambda^{-R}\right) d \eta_{0}\right\}=0
$$

and $\nu_{0}$ is the only non-singular measure $\eta_{0} \in \mathcal{M}_{S}$ which attains the supremum.

We first prove the following two lemmas. 
LEMMA 5.2. Let $\chi_{0}$ be the indicator function for $\Delta_{0}$. There exists a $k_{0} \in \mathbb{N}$ such that, for all $k \geq k_{0}$ and all $x \in \Delta_{0}$,

$$
\lambda^{-k} \varphi^{-1}(x) \mathcal{L}^{k}\left(\varphi \chi_{0}\right)(x) \geq v\left(\chi_{0}\right) / 2 .
$$

Proof. Note that $\chi_{0} \in \mathcal{B}_{0}$ so that $\lambda^{-k} \mathcal{L}^{k}\left(\chi_{0}\right) \rightarrow c\left(\chi_{0}\right) \varphi$ in the $\|\cdot\|$-norm. This means that the functions converge pointwise uniformly on each level of the tower. Thus

$$
0<v\left(\Delta_{0}\right)=\lim _{k \rightarrow \infty} \lambda^{-k} \varphi^{-1}(x) \mathcal{L}^{k}\left(\chi_{0} \varphi\right)(x)
$$

uniformly for $x \in \Delta_{0}$. The uniform convergence implies the existence of the desired $k_{0}$.

The next lemma establishes the Gibbs property for $\nu_{0}$.

LEMmA 5.3. Let $\left[i_{0}, i_{1}, \ldots, i_{n-1}\right] \subset \Delta_{0}$ denote a cylinder set of length $n$ with respect to $S$. Then there exists a constant $C>0$ such that for all $n$,

$$
C^{-1} \lambda^{-R_{n}\left(y_{*}\right)}\left(J S^{n}\left(y_{*}\right)\right)^{-1} \leq v\left(\left[i_{0}, i_{1}, \ldots, i_{n-1}\right]\right) \leq C \lambda^{-R_{n}\left(y_{*}\right)}\left(J S^{n}\left(y_{*}\right)\right)^{-1}
$$

where $y_{*}$ is an arbitrary point in $\left[i_{0}, i_{1}, \ldots, i_{n-1}\right]$ and $J S^{n}$ is the Jacobian of $S^{n}$ with respect to $m$.

Proof. Let $\chi_{A}$ be the indicator function of $A:=\left[i_{0}, i_{1}, \ldots, i_{n-1}\right]$. Although $\chi_{A} \notin \mathcal{B}$, we do have $\mathcal{L}^{k} \chi_{A} \in \mathcal{B}$ for $k \geq n$ since 1 -cylinders are in $\mathcal{B}$. Thus $v\left(\chi_{A}\right)$ is characterized by the limit $v\left(\chi_{A}\right)=\lim _{k} \lambda^{-k} \varphi^{-1} \mathcal{L}^{k}\left(\varphi \chi_{A}\right)$. Since this convergence is in the $\|\cdot\|$-norm, it is uniform for $x \in \Delta_{0}$.

For $x \in \Delta_{0}$ and $k \geq R_{n}(A)$,

$$
\begin{aligned}
\mathcal{L}^{k}\left(\varphi \chi_{A}\right)(x) & =\sum_{F^{k} y=x} \varphi(y) \chi_{A}(y) g_{k}(y) \\
& =\sum_{y \in A, F^{k} y=x} \varphi(y) g_{k-R_{n}(A)}\left(F^{R_{n}} y\right) g_{R_{n}}(y) \\
& =\sum_{z \in F^{R_{n}}(A), F^{k-R_{n}(A)} z=x} \varphi(y) g_{k-R_{n}(A)}(z) g_{R_{n}}(y),
\end{aligned}
$$

where in the last line we have used the fact that $\left.F^{R_{n}(A)}\right|_{A}$ is injective. Note that by (2.1), we may replace $g_{R_{n}}(y)$ by $g_{R_{n}}\left(y_{*}\right)$ where $y_{*} \in A$ is an arbitrary point. Also, since both $y$ and $F^{R_{n}} y$ are in $\Delta_{0}$ and $\delta \leq \varphi \leq \delta^{-1}$ on $\Delta_{0}$, we may estimate (5.1) by

$$
\begin{aligned}
\mathcal{L}^{k}\left(\varphi \chi_{A}\right)(x) & \leq C g_{R_{n}}\left(y_{*}\right) \sum_{z \in F^{R_{n}}(A), F^{k-R_{n}(A)} z=x} \varphi(z) g_{k-R_{n}(A)}(z) \\
& \leq C g_{R_{n}}\left(y_{*}\right) \sum_{F^{k-R_{n}(A)} z=x} \varphi(z) g_{k-R_{n}(A)}(z) \\
& =C g_{R_{n}}\left(y_{*}\right) \mathcal{L}^{k-R_{n}(A)} \varphi(x)=C g_{R_{n}}\left(y_{*}\right) \lambda^{k-R_{n}(A)} \varphi(x) .
\end{aligned}
$$

Combining this estimate with the definition of $v$ and noticing that $g_{R_{n}}=\left(J S^{n}\right)^{-1}$, we have the upper bound,

$$
v(A) \leq C\left(J S^{n}\left(y_{*}\right)\right)^{-1} \lambda^{-R_{n}(A)} .
$$


To obtain the lower bound, we again work from equation (5.1) and choose $k \geq R_{n}(A)+$ $n_{0}+k_{0}$.

$$
\begin{aligned}
& \mathcal{L}^{k}\left(\varphi \chi_{A}\right)(x)=\sum_{y \in A, F^{k} y=x} \varphi(y) g_{k-R_{n}(A)-n_{0}}\left(F^{R_{n}+n_{0}} y\right) g_{n_{0}}\left(F^{R_{n}} y\right) g_{R_{n}}(y) \\
& \geq \sum_{\substack{z \in F^{R_{n}+n_{0}}(A) \cap \Delta_{0} \\
F^{k-R_{n}(A)-n_{0}} z=x}} \varphi(y) g_{k-R_{n}(A)-n_{0}}(z) g_{n_{0}}\left(F^{R_{n}} y\right) g_{R_{n}}(y) .
\end{aligned}
$$

We again replace $g_{R_{n}}(y)$ by $g_{R_{n}}\left(y_{*}\right)$ using (2.1). Note also that $\left.g_{n_{0}}\right|_{\Delta_{0}}$ is bounded below and that $F^{R_{n}(A)} y \in \Delta_{0}$. Since we are only considering $y, z \in \Delta_{0}$, we know that $\varphi(y)$ is proportional to $\varphi(z)$. Thus

$$
\begin{aligned}
& \mathcal{L}^{k}\left(\varphi \chi_{A}\right)(x) \geq C g_{R_{n}}\left(y_{*}\right) \sum_{\substack{z \in F^{R n+n_{0}}(A) \cap \Delta_{0} \\
F^{k-R_{n}(A)-n_{0}} z=x}} \varphi(z) g_{k-R_{n}(A)-n_{0}}(z) \\
& =C g_{R_{n}}\left(y_{*}\right) \sum_{F^{k-R_{n}(A)-n_{0}} z=x} \chi_{0}(z) \varphi(z) g_{k-R_{n}(A)-n_{0}}(z) \\
& =C g_{R_{n}}\left(y_{*}\right) \mathcal{L}^{k-R_{n}(A)-n_{0}}\left(\chi_{0} \varphi\right)(x)
\end{aligned}
$$

where in the second to last line we have used the fact that $F^{R_{n}+n_{0}}(A) \supseteq \Delta_{0}$. Combining equation (5.4) with Lemma 5.2, since $k-R_{n}(A)-n_{0} \geq k_{0}$, we estimate

$$
\mathcal{L}^{k}\left(\varphi \chi_{A}\right)(x) \geq C g_{R_{n}}\left(y_{*}\right) \lambda^{k-R_{n}(A)-n_{0}} \varphi(x) \frac{v\left(\chi_{0}\right)}{2} .
$$

The lower bound follows from the definition of $v$.

Proof of Proposition 5.1. Lemma 5.3 implies that $v_{0}$ is a Gibbs measure with potential $\phi=-\log \left(\lambda^{R} J S\right)$. We define a topology on $\Delta$ using the cylinder sets with respect to $\mathcal{Z}$ as our basis. The fact that $\left.S\right|_{\Delta_{0} \cap \Delta^{\infty}}$ is topologically mixing follows immediately from the condition that $F$ be mixing on elements of $\mathcal{Z}_{0}^{i m}$ together with the finite images condition (P3). This can be seen as in the proof of Proposition 2.8(ii) in §3.3.

The formalism of $[\mathbf{S}]$ completes the proof of the proposition. [S, Theorem 3] implies that

$$
P_{G}(\phi)=\sup _{\eta_{0} \in \mathcal{M}_{S}}\left\{h_{\eta_{0}}(S)+\int_{\Delta_{0}} \phi d \eta_{0}\right\}
$$

where (with $P_{\text {top }}$ denoting the topological pressure)

$$
P_{G}(\phi)=\sup \left\{P_{\text {top }}\left(\left.\phi\right|_{Y}\right): Y \subset \Delta_{0} \cap \Delta^{\infty} \text {, topologically mixing finite Markov shift }\right\}
$$

is the Gurevich pressure of $\phi$ for $S$.

Lemma 5.3 of this paper combined with [S, Theorems 7 and 8] implies that $P_{G}(\phi)=0$ and that the supremum is obtained by our Gibbs measure $v_{0}$. In addition, $v_{0}$ is the only non-singular $S$-invariant probability measure which attains the supremum.

We now prove an equilibrium principle for $F$ using the one for $S$. 
LEMMA 5.4. Let $\mathcal{M}_{F}$ be the set of $F$-invariant Borel probability measures on $\Delta$. For any $\eta \in \mathcal{M}_{F}$, let $\eta_{0}=\left.\left(1 / \eta_{0}\left(\Delta_{0}\right)\right) \eta\right|_{\Delta_{0}}$. Then

$$
\int_{\Delta_{0}} \log J S d \eta_{0}=\int_{\Delta} \log J F d \eta \int_{\Delta_{0}} R d \eta_{0}
$$

Proof. Notice that $\eta_{0} \in \mathcal{M}_{S}$. For $x \in \Delta_{0}$,

$$
J S(x)=J F^{R}(x)=\prod_{i=0}^{R(x)-1} J F\left(F^{i} x\right) .
$$

However, $J F\left(F^{i} x\right)=1$ for $i<R(x)-1$, so that $J S(x)=J F\left(F^{R-1} x\right)$. In other words, we have

$$
\int_{\Delta_{0}} \log J S d \eta_{0}=\eta\left(\Delta_{0}\right)^{-1} \int_{F^{-1} \Delta_{0}} \log J F d \eta=\eta\left(\Delta_{0}\right)^{-1} \int_{\Delta} \log J F d \eta .
$$

Since the measure of a partition element $Z_{\ell, j} \in \mathcal{Z}$ does not change as it moves up the tower, we have

$$
1=\sum_{(\ell, j)} \eta\left(Z_{\ell, j}\right)=\sum_{j} \eta\left(Z_{0, j}\right) R\left(Z_{0, j}\right)=\int_{\Delta_{0}} R d \eta
$$

So by definition of $\eta_{0}$, we have

$$
\int_{\Delta_{0}} R d \eta_{0}=\eta\left(\Delta_{0}\right)^{-1} \int_{\Delta_{0}} R d \eta=\eta\left(\Delta_{0}\right)^{-1}
$$

This, together with (5.6), proves the lemma.

Since $S=F^{R}$ is a first return map to $\Delta_{0}$, the general formula of Abramov [A] implies that $h_{\eta}(F)=h_{\eta_{0}}(S) \eta\left(\Delta_{0}\right)$ so that

$$
h_{\eta_{0}}(S)=\eta\left(\Delta_{0}\right)^{-1} h_{\eta}(F)=h_{\eta}(F) \int_{\Delta_{0}} R d \eta_{0} .
$$

Since $\int_{\Delta_{0}} R d \eta_{0}=\eta\left(\Delta_{0}\right)^{-1} \neq 0$ and there is a one-to-one correspondence between measures in $\mathcal{M}_{S}$ and $\mathcal{M}_{F}$, putting equation (5.7) and Lemma 5.4 together with (5.5), we have

$$
\log \lambda=\sup _{\eta \in \mathcal{M}_{F}}\left\{h_{\eta}(F)-\int_{\Delta} \log J F d \eta\right\} .
$$

Moreover, $v$ is the only non-singular $F$-invariant probability measure which attains the supremum. This completes the proof of Theorem 2.9.

5.3. An equilibrium principle for $(T, X)$. The proof of Theorem 2.17 consists simply of projecting (5.8) down to $X$ to get the desired relation for $T$.

Note that for any $\eta \in \mathcal{M}_{F}$, we can define $\tilde{\eta}=\pi_{*} \eta \in \mathcal{M}_{T}$. Then given a function $\tilde{f}$ on $X$, we have $\int_{X} \tilde{f} d \tilde{\eta}=\int_{\Delta} \tilde{f} \circ \pi d \eta$. From the relation $\pi \circ F=T \circ \pi$, we have

$$
J \pi(F x) J F(x)=J T(\pi x) J \pi(x)
$$


for each $x \in \Delta$. Thus,

$$
\int_{X} \log J T d \tilde{\eta}=\int_{\Delta}(\log J F+\log J \pi \circ F-\log J \pi) d \eta=\int_{\Delta} \log J F d \eta
$$

since the last two terms cancel by the the $F$-invariance of $\eta$.

The fact that $h_{\eta}(F)=h_{\tilde{\eta}}(T)$ follows since $\pi$ is at most countable-to-one [Bu, Proposition 2.8]. Thus

$$
h_{\eta}(F)-\int_{\Delta} \log J F d \eta=h_{\tilde{\eta}}(T)-\int_{X} \log J T d \tilde{\eta}
$$

for each $\eta \in \mathcal{M}_{F}$, which proves the theorem.

Acknowledgements. HB was supported in part by EPSRC grant EP/F037112/1. MD was supported in part by EPSRC grant GR/S11862/01 and NSF grant DMS-0801139. IM was supported in part by EPSRC grant GR/S11862/01.

MD thanks the University of Surrey for an engaging visit during which this project was started. In addition, HB would like to thank Georgia Tech; MD would like to thank the Scuola Normale Superiore, Pisa; MD and IM would like to thank MSRI, Berkeley, where part of this work was done.

\section{REFERENCES}

[A] L. M. Abramov. The entropy of a derived automorphism. Dokl. Akad. Nauk. SSSR 128 (1959), 647-650. Amer. Math. Soc. Transl. Ser. 2 49(2) (1966), 162-166.

[Ba] V. Baladi. Positive Transfer Operators and Decay of Correlations (Advanced Series in Nonlinear Dynamics, 16). World Scientific, Singapore, 2000.

[BaK] V. Baladi and G. Keller. Zeta functions and transfer operators for piecewise monotonic transformations. Comm. Math. Phys. 127 (1990), 459-477.

[Bo] R. Bowen. Equilibrium States and the Ergodic Theory of Anosov Diffeomorphisms (Lecture Notes in Mathematics, 470). Springer, Berlin, 1975.

[BrK] H. Bruin and G. Keller. Equilibrium states for unimodal maps. Ergod. Th. \& Dynam. Sys. 18 (1998), 765-789.

[BLS] H. Bruin, S. Luzzatto and S. van Strien. Decay of correlations in one-dimensional dynamics. Ann. Sci. École Norm. Sup. (4) 36 (2003), 621-646.

[BT] H. Bruin and M. Todd. Equilibrium states for interval maps: the potential $-t \log |D f|$. Ann Sci. École Norm Sup. to appear.

[Bu] J. Buzzi. Markov extensions for multi-dimensional dynamical systems. Israel J. Math. 112 (1999), 357-380.

[C1] N. N. Cencova. A natural invariant measure on Smale's horseshoe. Soviet Math. Dokl. 23 (1981), 87-91.

[C2] N. N. Cencova. Statistical properties of smooth Smale horseshoes. Mathematical Problems of Statistical Mechanics and Dynamics. Ed. R. L. Dobrushin. Reidel, Dordrecht, 1986, pp. 199-256.

[CM1] N. Chernov and R. Markarian. Ergodic properties of Anosov maps with rectangular holes. Bol. Soc. Bras. Mat. 28 (1997), 271-314.

[CM2] N. Chernov and R. Markarian. Anosov maps with rectangular holes. Nonergodic cases. Bol. Soc. Bras. Mat. 28 (1997), 315-342.

[CMT1] N. Chernov, R. Markarian and S. Troubetzkoy. Conditionally invariant measures for Anosov maps with small holes. Ergod. Th. \& Dynam. Sys. 18 (1998), 1049-1073. 
[CMT2] N. Chernov, R. Markarian and S. Troubetzkoy. Invariant measures for Anosov maps with small holes. Ergod. Th. \& Dynam. Sys. 20 (2000), 1007-1044.

[CMS1] P. Collet, S. Martinez and B. Schmitt. The Yorke-Pianigiani measure and the asymptotic law on the limit Cantor set of expanding systems. Nonlinearity 7 (1994), 1437-1443.

[CMS2] P. Collet, S. Martinez and B. Schmitt. Quasi-stationary distribution and Gibbs measure of expanding systems. Instabilities and Nonequilibrium Structures V. Eds. E. Tirapegui and W. Zeller. Kluwer, Dordrecht, 1996, pp. 205-219.

[CV] N. Chernov and H. van den Bedem. Expanding maps of an interval with holes. Ergod. Th. \& Dynam. Sys. 22 (2002), 637-654.

[D1] M. Demers. Markov extensions for dynamical systems with holes: an application to expanding maps of the interval. Israel J. Math. 146 (2005), 189-221.

[D2] M. Demers. Markov extensions and conditionally invariant measures for certain logistic maps with small holes. Ergod. Th. \& Dynam. Sys. 25(4) (2005), 1139-1171.

[DL] M. Demers and C. Liverani. Stability of statistical properties in two-dimensional piecewise hyperbolic maps. Trans. Amer. Math. Soc. 360(9) (2008), 4777-4814.

[DY] M. Demers and L.-S. Young. Escape rates and conditionally invariant measures. Nonlinearity 19 (2006), 377-397.

[DHL] K. Díaz-Ordaz, M. Holland and S. Luzzatto. Statistical properties of one-dimensional maps with critical points and singularities. Stoch. Dyn. 6 (2006), 423-458.

[HY] A. Homburg and T. Young. Intermittency in families of unimodal maps. Ergod. Th. \& Dynam. Sys. 22(1) (2002), 203-225.

[K] G. Keller. Markov extensions, zeta-functions, and Fredholm theory for piecewise invertible dynamical systems. Trans. Amer. Math. Soc. 314 (1989), 433-497.

[Li] C. Liverani. Decay of correlations for piecewise expanding maps. J. Stat. Phys. 78 (1995), 1111-1129.

[LiM] C. Liverani and V. Maume-Deschamps. Lasota-Yorke maps with holes: conditionally invariant probability measures and invariant probability measures on the survivor set. Ann. Inst. H. Poincaré Probab. Statist. 39 (2003), 385-412.

[LoM] A. Lopes and R. Markarian. Open billiards: Cantor sets, invariant and conditionally invariant probabilities. SIAM J. Appl. Math. 56 (1996), 651-680.

[MS] W. de Melo and S. van Strien. One Dimensional Dynamics (Ergebnisse Series, 25). Springer, Berlin, 1993.

[PY] G. Pianigiani and J. Yorke. Expanding maps on sets which are almost invariant: decay and chaos. Trans. Amer. Math. Soc. 252 (1979), 351-366.

[R] P. A. Richardson Jr. Natural measures on the unstable and invariant manifolds of open billiard dynamical systems. Doctoral Dissertation, Department of Mathematics, University of North Texas, 1999.

[S] O. Sarig. Thermodynamic formalism of countable Markov shifts. Ergod. Th. \& Dynam. Sys. 19 (1999), 1565-1593.

[Y] L.-S. Young. Statistical properties of dynamical systems with some hyperbolicity. Ann. of Math. (2) 147 (1998), 585-650. 